\title{
Examples of exotic stratifications
}

\author{
BRUCE HUGHES \\ LAURENCE R TAYLOR \\ SHMUEL WEINBERGER \\ BRUCE WILliams
}

\begin{abstract}
We produce examples of manifold stratified pairs in which the lower strata do not have neighborhoods that are mapping cylinders of fiber bundles, or even block bundles. Moreover, the examples do not improve in this regard under stabilization by products with tori. The examples are locally conelike and the lower strata do have neighborhoods which are mapping cylinders of manifold approximate fibrations. They are constructed by combining the classification of manifold approximate fibrations with the authors' classification of neighborhood germs.
\end{abstract}

57N80; 19J99, 55R65, 57N55

\section{Introduction}

This paper is about the glue that holds together stratified spaces. Stratified spaces are spaces that are made up out of manifold pieces; that is, one has a space $X$ that is decomposed into subsets, each of which is an open manifold and which "fit together nicely." The basic examples one has in mind are algebraic varieties and quotients of group actions, but it is quite reasonable and occasionally useful to think of manifolds with boundary or manifolds together with embedded (or immersed) submanifolds as examples as well.

But, while one might have mental pictures of these examples, to some extent, just saying "quotient of a group action" or "embedded submanifold" really begs the geometric question. What kind of regularity shall we assume that the action or the embedding has? The theory of Whitney stratified spaces (see, eg Whitney [44], Thom [38] and Mather [28]) is based on modeling on the theory of smooth embedding and smooth group actions, where one has a good bundle neighborhood (according to the tubular neighborhood theorem). One assumes that each open manifold stratum in $X$ has a neighborhood, which is given a bundle structure.

For instance, even nice locally flat topological manifolds do not necessarily have bundle structures. A theory adequate for (and doubtlessly modeled on) PL topology was 
introduced by Browder and Quinn [7], and is quite similar to the Whitney theory, but essentially replaces the bundles by block bundles. We will call this an sss = strong stratification structure. However, even this is not enough for topological applications: even if one assumes that a group action is locally linear (ie each orbit has an invariant neighborhood which is equivariantly homeomorphic to an open subset of a representation space), the quotient space need not have an sss. Moreover, ssss are not unique: had they been, there would be no possibility for the celebrated phenomenon, discovered by Cappell and Shaneson [8], of nonlinear similarity of linear representations; DeRham's original proof that linear representation spheres are not PL conjugate only made use of the possibility of deleting in a well defined fashion open regular neighborhoods of strata. Ssss enable one to have a completely straightforward theory of Whitehead torsion, and $h$-cobordism.

In this paper we shall be interested in weakly stratified (or homotopically stratified) spaces, defined initially by Quinn [32], although our examples will also be CS spaces of Siebenmann [36]. Both of these are nice topologically invariant notions, but the latter are a bit less flexible than the former. These spaces are as topologically homogeneous as one could hope: any two points in the same component of a pure stratum can be moved to one another by a homeomorphism of $X$ isotopic to the identity. Typically, any class of space defined by a local homeomorphism condition will sit in the framework, but often compactifications of covers and similar "wild" constructions will also have enough homological and homotopical control to fit into, at least, Quinn's framework.

The work of Anderson and Hsiang [2; 4] (which predated Quinn's definition, and hence directly addresses triangulation) shows that, in some sense, the whole difference between these theories can be attributed to the algebraic $K$-theory of the fundamental groups of various links of strata in one another. Thus, for instance, in the situation of locally flat embeddings, an sss does exist and is unique, because $\mathrm{Wh}_{1}(e)=0=\widetilde{K}_{n}(e)$ for $n<1$.

It is a general yoga that algebraic $K$-theory obstructions tend to die when one takes products with a circle; if there is a series of them one could imagine the need to take a product of several circles: after crossing with a circle, $h$-cobordisms become products, finitely dominated complexes become finite, open manifolds with tame ends can be given (canonical) boundaries, and at the cost of using a number of circles, block bundles become bundles (see Weiss and Williams [43]). The main result of this paper is that even for the very simplest stratified spaces with just two strata, this fails for the issue of a finding an sss.

Main Theorem For every $m>5$, there is a locally conelike stratified space $X$ of dimension $m$, with singular set a circle, and which has no sss (and certainly no Whitney 
stratification) even after crossing with any torus. Moreover, $X$ can be chosen so that the singular circle possesses a mapping cylinder neighborhood.

It follows from the theory of Hughes, Taylor, Weinberger and Williams [18] that there is no manifold $M$ so that $M \times X$ has an sss. Our proof combines the teardrop neighborhood theorem of [18], with the classification of approximate fibrations from Hughes, Taylor and Williams [19], the classification of bounded concordances from Anderson and Hsiang [3; 4], known calculations of Whitehead and $K$-groups, and results of T Lawson on inertial $h$-cobordisms [26].

A different approach to these results could be obtained by analogy to the theory of Rothenberg characteristic classes from Cappell and Weinberger [9]; these were defined in the PL context and measured the obstruction to concording a PL action with manifold fixed point set to one which is PL homogeneous: the former all have homotopy stratified structures and the latter are all sss. They take values in the (ordinary!) cohomology of the fixed set with coefficients in the Tate cohomology of the group acting. In our setting, the classes would be associated to the Tate cohomology of a truncated (nonconnective) Whitehead spectrum associated to the fundamental group of the holink. Whatever advantages there might be to such a development, brevity is not one of them; we hope that the current treatment is both more direct and more broadly accessible.

This paper is organized as follows. The Anderson-Hsiang theory and its relation to inertial $h$-cobordisms are recalled in Section 2. The theory of controlled homeomorphisms and the Hughes-Taylor-Williams manifold approximate fibration classification are discussed in Section 3. That section also explains how the Hughes-Taylor-Williams classification interacts with the classical classification of fiber bundles and the special form that those classifications take on when the base is the circle $S^{1}$. The classifications are combined with known calculations in Section 4 in order to produce exotic manifold approximate fibrations over $S^{1}$; that is, manifold approximate fibrations that are not controlled homeomorphic to fiber bundles even after euclidean stabilization. Finally, these exotic manifold approximate fibrations are combined with the Hughes-TaylorWeinberger-Williams neighborhood germ classification in Section 5 in order to produce the examples in the Main Theorem above.

\section{Acknowledgements}

The first author was supported in part by NSF Grant DMS-9971367. The second and fourth authors were supported in part by NSF Grant DMS-0204169. The third author was supported in part by NSF Grant DMS-0204615. 


\section{Anderson-Hsiang theory and inertial $h$-cobordisms}

In this section we recall the theory of Anderson and Hsiang [3; 4] that relates bounded concordances and bounded homeomorphisms, and give their calculation of the components of the space of bounded concordances. This is important for us because manifold approximate fibrations are classified by bounded homeomorphisms (see Section 3) and neighborhood germs are classified by manifold approximate fibrations (see Section 5). This section also contains a purely algebraic fact (Lemma 2.6) about interlocking exact sequences that we will encounter.

For the remainder of this section, let $F$ denote a closed connected manifold of dimension $n$. Let $\operatorname{TOP}^{\mathrm{b}}\left(F \times \mathbb{R}^{i}\right)$ denote the simplicial set of bounded homeomorphisms on $F \times \mathbb{R}^{i}$ so that a $k$-simplex of $\operatorname{TOP}^{\mathrm{b}}\left(F \times \mathbb{R}^{i}\right)$ consists of a homeomorphism $h: F \times \mathbb{R}^{i} \times \Delta^{k} \rightarrow$ $F \times \mathbb{R}^{i} \times \Delta^{k}$ such that $h$ is fiber preserving over $\Delta^{k}$ and bounded in the $\mathbb{R}^{i}$-direction. This latter condition means there exists a constant $c>0$ such that $p_{2} h$ is $c$-close to $p_{2}$ where $p_{2}: F \times \mathbb{R}^{i} \times \Delta^{k} \rightarrow \mathbb{R}^{i}$ is projection.

Let $\mathrm{C}^{\mathrm{b}}\left(F \times \mathbb{R}^{i}\right)$ denote the simplicial set of bounded concordances on $F \times \mathbb{R}^{i}$ so that a $k$-simplex of $\mathrm{C}^{\mathrm{b}}\left(F \times \mathbb{R}^{i}\right)$ consists of a homeomorphism $h: F \times \mathbb{R}^{i} \times[0,1] \times \Delta^{k} \rightarrow$ $F \times \mathbb{R}^{i} \times[0,1] \times \Delta^{k}$ such that $h$ is fiber preserving over $\Delta^{k}, h \mid: F \times \mathbb{R}^{i} \times\{0\} \times \Delta^{k} \rightarrow$ $F \times \mathbb{R}^{i} \times\{0\} \times \Delta^{k}$ is the identity, and $h$ is bounded over $\mathbb{R}^{i}$.

A bounded concordance on $F \times \mathbb{R}^{i}$ induces a bounded homeomorphism on $F \times \mathbb{R}^{i}$ by restricting the concordance to $F \times \mathbb{R}^{i} \times\{1\}$. This defines a simplicial map

$$
\rho: \mathrm{C}^{\mathrm{b}}\left(F \times \mathbb{R}^{i}\right) \rightarrow \operatorname{TOP}^{\mathrm{b}}\left(F \times \mathbb{R}^{i}\right)
$$

by setting $\rho(h)=h \mid: F \times \mathbb{R}^{i} \times\{1\} \times \Delta^{k}=F \times \mathbb{R}^{i} \times \Delta^{k} \rightarrow F \times \mathbb{R}^{i} \times\{1\} \times \Delta^{k}=F \times \mathbb{R}^{i} \times \Delta^{k}$.

Euclidean stabilization induces a simplicial map

$$
\sigma: \operatorname{TOP}^{\mathrm{b}}\left(F \times \mathbb{R}^{i}\right) \rightarrow \operatorname{TOP}^{\mathrm{b}}\left(F \times \mathbb{R}^{i+1}\right) ; \quad h \mapsto h \times \mathrm{id}_{\mathbb{R}}
$$

and, in particular, a group homomorphism $\pi_{0} \operatorname{TOP}^{\mathrm{b}}\left(F \times \mathbb{R}^{i}\right) \stackrel{\sigma}{\rightarrow} \pi_{0} \mathrm{TOP}^{\mathrm{b}}\left(F \times \mathbb{R}^{i+1}\right)$ for each $i \geq 0$.

Proposition 2.1 (Anderson and Hsiang) There is a homotopy fibration sequence

$$
\mathrm{C}^{\mathrm{b}}\left(F \times \mathbb{R}^{i}\right) \stackrel{\rho}{\rightarrow} \operatorname{TOP}^{\mathrm{b}}\left(F \times \mathbb{R}^{i}\right) \stackrel{\sigma}{\rightarrow} \operatorname{TOP}^{\mathrm{b}}\left(F \times \mathbb{R}^{i+1}\right) .
$$

In particular, there is a short exact sequence

$$
\pi_{0} \mathrm{C}^{\mathrm{b}}\left(F \times \mathbb{R}^{i}\right) \stackrel{\rho}{\rightarrow} \pi_{0} \mathrm{TOP}^{\mathrm{b}}\left(F \times \mathbb{R}^{i}\right) \stackrel{\sigma}{\rightarrow} \pi_{0} \mathrm{TOP}^{\mathrm{b}}\left(F \times \mathbb{R}^{i+1}\right) .
$$


Proof This is essentially the fibration of Anderson and Hsiang [4, Lemma 9.3]. One must use Anderson and Hsiang [3, Theorem 4] to identify $\mathrm{C}^{\mathrm{b}}\left(F \times \mathbb{R}^{i}\right)$ with the fiber in [4]. Similarly one needs a reinterpretation of $\operatorname{TOP}^{\mathrm{b}}\left(F \times \mathbb{R}^{i}\right)$. See Hughes, Taylor and Williams [21, Theorem 1.2] for an explicit proof of the reinterpretation. See also Lashof and Rothenberg [25, Section 8].

An inertial $h$-cobordism on $F$ is an $h$-cobordism $\left(W ; \partial_{0} W, \partial_{1} W\right)$ with $\partial_{0} W=$ $F$ and $\partial_{1} W$ homeomorphic to $F$. It is possible to define the simplicial set of $h-$ cobordisms on $F$ (eg Waldhausen [39]) and the simplicial set of inertial $h$-cobordisms on $F$. However, for this paper we only need the sets of components of these simplicial sets. Thus, let $\pi_{0} h \operatorname{cob}(F)$ denote the set of equivalence classes of $h$-cobordisms on $F$ such that $\left(W ; \partial_{0} W, \partial_{1} W\right)$ is equivalent to $\left(W^{\prime} ; \partial_{0} W^{\prime}, \partial_{1} W^{\prime}\right)$ if and only if there exists a homeomorphism $H: W \rightarrow W^{\prime}$ such that $H \mid: \partial_{0} W=F \rightarrow \partial_{0} W^{\prime}=F$ is the identity. The set $\pi_{0} \mathrm{I} h \operatorname{cob}(F)$ of inertial $h$-cobordisms on $F$ is the subset of $\pi_{0} h \operatorname{cob}(F)$ consisting of all classes represented by inertial $h$-cobordisms.

The $s$-cobordism theorem gives a bijection

$$
\pi_{0} h \operatorname{cob}(F) \stackrel{\tau}{\rightarrow} \mathrm{Wh}_{1}\left(\mathbb{Z} \pi_{1} F\right)
$$

provided $n \geq 5$, which sends an $h$-cobordism $\left(W ; \partial_{0} W, \partial_{1} W\right)$ to the Whitehead torsion $\tau\left(W, \partial_{0} W\right)$ in $\mathrm{Wh}_{1}\left(\mathbb{Z} \pi_{1} F\right)$. In general, the image of $\pi_{0} \mathrm{I} h \operatorname{cob}(F)$ in $\mathrm{Wh}_{1}\left(\mathbb{Z} \pi_{1} F\right)$ need not be a subgroup (cf Hausmann [16], Ling [27]).

We now recall the well-known region between construction (cf Anderson and Hsiang [3, Section 8]) which defines a function

$$
\beta: \pi_{0} \mathrm{TOP}^{\mathrm{b}}(F \times \mathbb{R}) \longrightarrow \pi_{0} \mathrm{I} h \operatorname{cob}(F) .
$$

If $h: F \times \mathbb{R} \rightarrow F \times \mathbb{R}$ is a bounded homeomorphism representing a class

$$
[h] \in \pi_{0} \mathrm{TOP}^{\mathrm{b}}(F \times \mathbb{R}),
$$

choose a $L>0$ so large that $h(F \times\{L\}) \subseteq F \times(0, \infty)$. Let $W=h(F \times(-\infty, L]) \backslash F \times$ $(-\infty, 0), \partial_{0} W=F \times\{0\}=F$, and $\partial_{1} W=h(F \times\{L\})$. Then $\left(W ; \partial_{0} W, \partial_{1} W\right)$ is an inertial $h$-cobordism on $F$ representing a class $[W] \in \pi_{0} \mathrm{I} h \operatorname{cob}(F)$. Set $\beta([h])=[W]$. The function $\beta$ is well-defined by the Isotopy Extension Theorem of Edwards and Kirby [11]. One should not confuse $\tau(\beta(h))$ with the torsion of the homotopy equivalence

$$
h_{1}: F=F \times\{0\} \hookrightarrow F \times \mathbb{R} \stackrel{h}{\rightarrow} F \times \mathbb{R} \stackrel{\text { proj }}{\longrightarrow} F .
$$

To see the relationship between these two torsions let $j: W \rightarrow[0,1]$ be any map with $j^{-1}(0)=\partial_{0} W$ and $j^{-1}(1)=\partial_{1} W$. Since $F \times\{0\} \hookrightarrow F \times(-\infty, 0]$ and 
$h(F \times\{L\}) \hookrightarrow h(F \times[L,+\infty))$ are homotopy equivalences, so is the inclusion $i: W \hookrightarrow F \times \mathbb{R}$, and there is a homotopy equivalence of triads

$$
\gamma=(\operatorname{proj} \circ i) \times j:\left(W ; \partial_{0} W, \partial_{1} W\right) \rightarrow(F \times[0,1] ; F \times\{0\}, F \times\{1\}) .
$$

Therefore,

$$
\tau\left(h_{1}\right)=\tau\left(\gamma \mid \partial_{1} W: \partial_{1} W \rightarrow F \times\{1\}\right)=\tau \beta(h)-(-1)^{n} \overline{\tau \beta(h)} \in \mathrm{Wh}_{1}\left(\mathbb{Z} \pi_{1} F\right),
$$

where - is induced from the standard involution on $\mathbb{Z} \pi_{1} F$. Although the composition

$$
\pi_{0} \mathrm{TOP}^{\mathrm{b}}(F \times \mathbb{R}) \stackrel{\beta}{\rightarrow} \pi_{0} \mathrm{I} h \operatorname{cob}(F) \stackrel{\tau}{\rightarrow} \mathrm{Wh}_{1}\left(\mathbb{Z} \pi_{1} F\right)
$$

need not be a group homomorphism (cf Ling [27]), it is a crossed homomorphism; ie, $\tau \beta([h \circ k])=h_{1 \sharp} \tau \beta([k])+\tau \beta([h])$ for $[h],[k] \in \pi_{0} \operatorname{TOP}^{\mathrm{b}}(F \times \mathbb{R})$ where $h_{1 \sharp}$ is the homomorphism induced by the homotopy equivalence $h_{1}: F \rightarrow F$.

We will need the following version of the Alexander trick in the proof of Proposition 2.3 (cf Hughes [17, Lemma 6.4]).

Lemma 2.2 If $h: F \times \mathbb{R} \rightarrow F \times \mathbb{R}$ is a bounded homeomorphism such that $h=\mathrm{id}$ on $F \times(-\infty, 0]$, then $h$ is boundedly isotopic to $\operatorname{id}_{F \times \mathbb{R}}$.

Proof For $0 \leq s<1$ define $\theta_{s}: \mathbb{R} \rightarrow \mathbb{R}$ by $\theta_{s}(t)=t-\frac{s}{s-1}$. Define a bounded isotopy $H: h \simeq \mathrm{id}_{F \times \mathbb{R}}$ by

$$
H_{S}= \begin{cases}\left(\operatorname{id}_{F} \times \theta_{S}\right)^{-1} \circ h \circ\left(\operatorname{id}_{F} \times \theta_{S}\right) & \text { if } 0 \leq s<1 \\ \operatorname{id}_{F \times \mathbb{R}} & \text { if } s=1 .\end{cases}
$$

Proposition 2.3 If $n=\operatorname{dim} F \geq 4$, then the sequence

$$
\pi_{0} \mathrm{TOP}(F) \stackrel{\sigma}{\rightarrow} \pi_{0} \mathrm{TOP}^{\mathrm{b}}(F \times \mathbb{R}) \stackrel{\beta}{\rightarrow} \pi_{0} \mathrm{I} h \operatorname{cob}(F) \longrightarrow 0
$$

is exact in the sense that $\beta$ maps the set of cosets of $\pi_{0} \mathrm{TOP}^{\mathrm{b}}(F \times \mathbb{R}) / \operatorname{Im}(\sigma)$ bijectively onto $\pi_{0} \mathrm{I} h \operatorname{cob}(F)$; ie

(i) if $\left[h_{1}\right],\left[h_{2}\right] \in \pi_{0} \mathrm{TOP}^{\mathrm{b}}(F \times \mathbb{R})$, then $\beta\left(\left[h_{1}\right]\right)=\beta\left(\left[h_{2}\right]\right)$ if and only if there exists $[g] \in \pi_{0} \operatorname{TOP}(F)$ such that $\left[h_{2}^{-1} h_{1}\right]=\sigma([g])$, and

(ii) $\beta$ is surjective.

Proof (i) Let $h_{i}: F \times \mathbb{R} \rightarrow F \times \mathbb{R}$ be bounded homeomorphisms for $i=1$, 2. Choose $L>0$ such that $h_{i}(F \times\{L\}) \subseteq F \times(0, \infty)$ so that $W_{i}=h_{i}(F \times(-\infty, L]) \backslash F \times(-\infty, 0)$ is an $h$-cobordism from $F=F \times\{0\}$ to $h_{i}(F \times\{L\})$ and $\beta\left(\left[h_{i}\right]\right)=\left[W_{i}\right] \in \pi_{0} h \operatorname{cob}(F)$ for $i=1,2$. If $\left[W_{1}\right]=\left[W_{2}\right]$, then there exists a homeomorphism $H: W_{1} \rightarrow W_{2}$ such that 
$H \mid: F \times\{0\} \rightarrow F \times\{0\}$ is the identity. In particular, $H h_{1}(F \times\{1\})=h_{2}(F \times\{L\})$. Let $g: F \rightarrow F$ be the homeomorphism defined by $h_{2}^{-1} H h_{1}(x, L)=(g(x), 1) \in F \times\{L\}$ for all $x \in F$. Extend $H$ via the identity on $F \times(-\infty, 0]$ to a homeomorphism $\widetilde{H}:(F \times(-\infty, 0]) \cup W_{1} \rightarrow(F \times(-\infty, 0]) \cup W_{2}$. Define a hybrid homeomorphism $\widetilde{h}: F \times \mathbb{R} \rightarrow F \times \mathbb{R}$ by

$$
\widetilde{h}(x, t)= \begin{cases}\widetilde{H} h_{1}(x, t) & \text { if } t \leq L \\ h_{2}(g(x), t) & \text { if } t \geq L .\end{cases}
$$

According to Lemma 2.2 both $\widetilde{h} h_{1}^{-1}$ and $\widetilde{h}\left(g^{-1} \times \mathrm{id}_{\mathbb{R}}\right) h_{2}^{-1}$ are boundedly isotopic to the identity. Thus $\widetilde{h}$ is boundedly isotopic to $h_{1}$ and to $h_{2}\left(g \times \mathrm{id}_{\mathbb{R}}\right)$ so that $h_{2}^{-1} h_{1}$ is boundedly isotopic to $g \times \operatorname{id}_{\mathbb{R}}$ showing $\left[h_{2}^{-1} h_{1}\right]=\sigma([g])$.

Conversely, if $h_{2}^{-1} h_{1}$ is boundedly isotopic to $g \times \mathrm{id}_{\mathbb{R}}$ for some homeomorphism $g: F \rightarrow F$, then $h_{1}$ is boundedly isotopic to $h_{2}\left(g \times \mathrm{id}_{\mathbb{R}}\right)$. If $L$ is large enough, then the isotopy restricts to an isotopy of embeddings carrying $h_{1}(F \times\{L\})$ onto $h_{2}(g(F) \times\{L\})=h_{2}(F \times\{L\})$ in $F \times(0, \infty)$. The Isotopy Extension Theorem [11] shows that there is an isotopy of $F \times \mathbb{R}$ to itself which is the identity on $F \times(-\infty, 0]$ and carries $h_{1}(F \times\{L\})$ to $h_{2}(F \times\{L\})$. In particular, there is a homeomorphism $H: W_{1} \rightarrow W_{2}$ such that $H \mid F \times\{0\}$ is the identity. Hence $\left[W_{1}\right]=\left[W_{2}\right] \in \pi_{0} h \operatorname{cob}(F)$.

(ii) follows from Ling [27, Proposition 3.2].

Anderson and Hsiang [3] calculated the homotopy groups of the simplicial set of bounded concordances. We will need their calculation of the group of components.

Proposition 2.4 (Anderson-Hsiang) If $n=\operatorname{dim} F \geq 5$, then there exists a group isomorphism

$$
\alpha: \pi_{0} \mathrm{C}^{\mathrm{b}}\left(F \times \mathbb{R}^{i}\right) \longrightarrow \begin{cases}\mathrm{Wh}_{1}\left(\mathbb{Z} \pi_{1} F\right) & \text { if } i=1 \\ \widetilde{K}_{0}\left(\mathbb{Z} \pi_{1} F\right) & \text { if } i=2 \\ K_{2-i}\left(\mathbb{Z} \pi_{1} F\right) & \text { if } i>2\end{cases}
$$

We need to recall the explicit construction of the isomorphism when $i=1$,

$$
\alpha: \pi_{0} \mathrm{C}^{\mathrm{b}}(F \times \mathbb{R}) \rightarrow \mathrm{Wh}_{1}\left(\mathbb{Z} \pi_{1} F\right) .
$$

If $h: F \times \mathbb{R} \times[0,1] \rightarrow F \times \mathbb{R} \times[0,1]$ is a bounded concordance representing a class $[h] \in \pi_{0} \mathrm{C}^{\mathrm{b}}(F \times \mathbb{R})$, choose $L>0$ so large that $h(F \times\{L\} \times[0,1]) \subseteq F \times(0, \infty) \times[0,1]$ and let

$$
W=h(F \times(-\infty, L] \times[0,1]) \backslash F \times(-\infty, 0) \times[0,1],
$$


$\partial_{0} W=F \times[0, L] \times\{0\}$, and $\partial_{1} W=h(F \times(-\infty, L] \times\{1\}) \backslash F \times(-\infty, 0) \times\{1\}$. Then $\left(W ; \partial_{0} W, \partial_{1} W\right)$ is a relative $h$-cobordism. In particular, over the boundary of $\partial_{0} W$, $W$ restricts to a product $h$-cobordism

$(F \times\{0\} \times[0,1] \cup h(F \times\{L\} \times[0,1]) ; F \times\{0, L\} \times\{0\}, F \times\{0\} \times\{1\} \cup h(F \times\{L\} \times\{1\}))$.

Define $\alpha([h])$ to be the Whitehead torsion $\tau\left(W, \partial_{0} W\right) \in \mathrm{Wh}_{1}\left(\mathbb{Z} \pi_{1}(F \times[0, L])\right)=$ $\mathrm{Wh}_{1}\left(\mathbb{Z} \pi_{1} F\right)$.

Recall that $\operatorname{dim} F=n$. Define the norm homomorphism

$$
N: \mathrm{Wh}_{1}\left(\mathbb{Z} \pi_{1} F\right) \rightarrow \mathrm{Wh}_{1}\left(\mathbb{Z} \pi_{1} F\right) ; \quad x \mapsto x+(-1)^{n} \bar{x}
$$

where $\leftarrow$ is induced from the standard involution on $\mathbb{Z} \pi_{1} F$.

Proposition 2.5 If $n=\operatorname{dim} F \geq 5$, then the following diagram commutes:

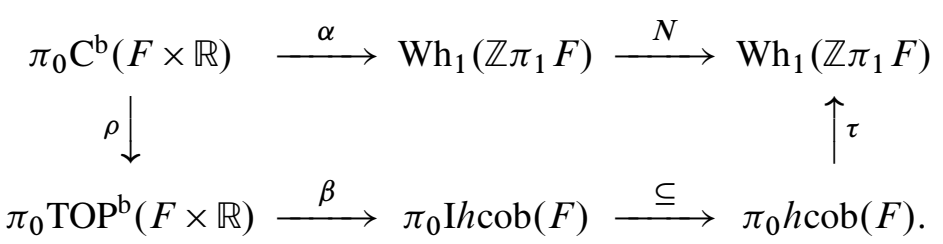

Proof If $[h] \in \pi_{0} \mathrm{C}^{\mathrm{b}}(F \times \mathbb{R})$ adopt the notation above in the explicit description of $\alpha$ so that $\alpha([h])=\tau\left(W, \partial_{0} W\right)=x$. For $k=0,1$ let $i_{k}: \partial_{k} W \rightarrow W$ denote the inclusion and $r_{k}: W \rightarrow \partial_{k} W$ a strong deformation retraction. Then $x=\tau\left(r_{0}\right)$. Since $\left(W ; \partial_{0} W, \partial_{1} W\right)$ is a relative $h$-cobordism between $(n+1)$-dimensional manifolds, it follows that $\left(r_{0} i_{1}\right)_{*} \tau\left(r_{1}\right)=\left(r_{0} i_{1}\right)_{*} \tau\left(W, \partial_{1} W\right)=(-1)^{n+1} \bar{x}$ by the duality theorem of Milnor [29, page 394]. Thus, $\tau\left(i_{1}\right)=(-1)^{n+1} \bar{\tau}\left(i_{0}\right) \in \mathrm{Wh}_{1}\left(\mathbb{Z} \pi_{1} W\right)$.

Let $j_{1}: F \times\{0\} \times\{1\} \rightarrow W$ and $j_{2}: F \times\{0\} \times\{1\} \rightarrow \partial_{0} W$ denote the inclusions and let $j_{3}: F \times\{0\} \times\{1\} \rightarrow \partial_{0} W$ be the map $j_{3}(z, 0,1)=(z, 0,0)$. Since $\partial_{0} W=$ $F \times[0, L] \times\{0\}, \tau\left(j_{3}\right)=0$.

Since $\tau \beta \rho([h])$ is the Whitehead torsion of $\left(\partial_{1} W, F \times\{0\} \times\{1\}\right)$ in $\mathrm{Wh}_{1}\left(\mathbb{Z} \pi_{1} F\right)$, it suffices to show that

$$
i_{1 *} \tau\left(j_{2}\right)=\tau\left(i_{0}\right)=(-1)^{n} \bar{\tau}\left(i_{0}\right) \in \mathrm{Wh}_{1}\left(\mathbb{Z} \pi_{1} W\right) .
$$

The composition formula gives

$$
\tau\left(j_{1}\right)=\tau\left(i_{1} j_{2}\right)=i_{1 *} \tau\left(j_{2}\right)+\tau\left(i_{1}\right) .
$$

Since $j_{1} \simeq i_{0} j_{3}$ and $\tau\left(j_{3}\right)=0$, the composition formula also gives $\tau\left(j_{1}\right)=\tau\left(i_{0} j_{3}\right)=$ $\tau\left(i_{0}\right)$. Thus

$$
i_{1 *} \tau\left(j_{2}\right)=\tau\left(i_{0}\right)-\tau\left(i_{1}\right)=\tau\left(i_{0}\right)+(-1)^{n+1} \tau\left(i_{0}\right) .
$$


A similar argument has been used by Siebenmann and Sondow [37, page 266].

Lemma 2.6 (i) Suppose there is a diagram

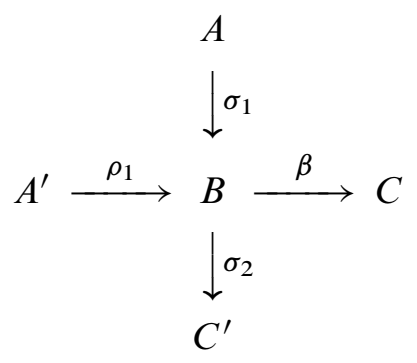

such that

(1) $A, B, A^{\prime}, C^{\prime}$ are groups (written additively) $C$ is a set, and $\sigma_{1}, \rho_{1}, \sigma_{2}$ are group homomorphisms,

(2) $A \stackrel{\sigma_{1}}{\rightarrow} B \stackrel{\beta}{\rightarrow} C$ is exact in the sense that $\beta$ is surjective, and if $b_{1}, b_{2} \in B$ then $\beta\left(b_{1}\right)=\beta\left(b_{2}\right)$ if and only if $b_{2}-b_{1}=\sigma_{1}(a)$ for some $a \in A$,

(3) $A^{\prime} \stackrel{\rho_{1}}{\longrightarrow} B \stackrel{\sigma_{2}}{\longrightarrow} C^{\prime}$ is an exact sequence of groups.

If $b \in B$, then $\sigma_{2}(b) \in \operatorname{Im}\left(\sigma_{2} \sigma_{1}: A \rightarrow C^{\prime}\right)$ if and only if $\beta(b) \in \operatorname{Im}\left(\beta \rho_{1}: A^{\prime} \rightarrow C\right)$.

(ii) Suppose further that the diagram above is extended to a diagram

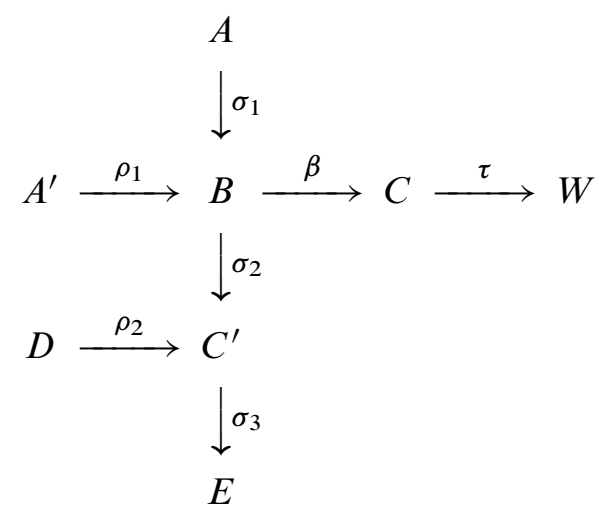

such that

(1) $A, B, A^{\prime}, C^{\prime}, W, D, E$ are abelian groups and $\rho_{2}, \sigma_{3}$ are group homomorphisms,

(2) $\tau: C \rightarrow W$ is a set inclusion,

(3) there is a $B$-module structure on $W$ which satisfies: if $b_{1}, b_{2} \in B$ and $\sigma_{2}\left(b_{1}\right)=$ $\sigma_{2}\left(b_{2}\right)$, then $b_{1} w=b_{2} w$ for all $w \in W$, 
(4) $\tau \beta: B \rightarrow W$ is a crossed homomorphism with respect to the $B$-module structure (ie $\tau \beta\left(b_{1}+b_{2}\right)=b_{1} \tau \beta\left(b_{2}\right)+\tau \beta\left(b_{1}\right)$ for all $b_{1}, b_{2} \in B$ ),

(5) $\tau \beta(-b)=-\tau \beta(b)$ for all $b \in B$,

(6) $N=\tau \beta \rho_{1}: A^{\prime} \rightarrow W$ is a homomorphism,

(7) $D \stackrel{\rho_{2}}{\longrightarrow} C^{\prime} \stackrel{\sigma_{3}}{\longrightarrow} E$ is an exact sequence of groups.

There exists a function $\widetilde{\beta}: \operatorname{Im}\left(\sigma_{2}\right) \rightarrow W / \operatorname{Im}(N)$ such that if $b \in B$, then $\sigma_{3} \sigma_{2}(b) \in$ $\operatorname{Im}\left(\sigma_{3} \sigma_{2} \sigma_{1}\right)$ if and only if the class of $\tau \beta(b)$ in $W / \operatorname{Im}(N)$ is in $\widetilde{\beta}\left[\operatorname{Im}\left(\sigma_{2}\right) \cap \operatorname{Im}\left(\rho_{2}\right)\right]$.

Proof (i) Suppose first that $\sigma_{2}(b)=\sigma_{2} \sigma_{1}(a)$ for some $a \in A$. Then the exact sequence of groups implies that there exists $a^{\prime} \in A^{\prime}$ such that $\rho_{1}\left(a^{\prime}\right)=b+\sigma_{1}(-a)$. Thus $-b+\rho_{1}\left(a^{\prime}\right)=\sigma_{1}(-a)$ and exactness of the other sequence implies $\beta(b)=\beta \rho_{1}\left(a^{\prime}\right)$.

Conversely, suppose $\beta(b)=\beta \rho_{1}\left(a^{\prime}\right)$ for some $a^{\prime} \in A^{\prime}$. Exactness implies that $b=$ $\sigma_{1}(a)+\rho_{1}\left(a^{\prime}\right)$ for some $a \in A$. Thus $\sigma_{2}(b)=\sigma_{2} \sigma_{1}(a)+\sigma_{2} \rho_{1}\left(a^{\prime}\right)=\sigma_{2} \sigma_{1}(a)$.

(ii) Define $\widetilde{\beta}: \operatorname{Im}\left(\sigma_{2}\right) \rightarrow W / \operatorname{Im}(N)$ by $\widetilde{\beta}(x)=\tau \beta \sigma_{2}^{-1}(x)$. In order to show that $\widetilde{\beta}$ is well-defined, suppose that $\sigma_{2}\left(y_{1}\right)=\sigma_{2}\left(y_{2}\right)$ and show that $\tau \beta\left(y_{1}\right)-\tau \beta\left(y_{2}\right) \in \operatorname{Im}(N)$. Since $\operatorname{ker}\left(\sigma_{2}\right)=\operatorname{Im}\left(\rho_{1}\right)$, it follows that $\tau \beta\left(y_{1}-y_{2}\right) \in \operatorname{Im}(N)$. Now

$$
\begin{gathered}
\tau \beta\left(y_{1}-y_{2}\right)-\left[\tau \beta\left(y_{1}\right)-\tau \beta\left(y_{2}\right)\right]=\left(-y_{2}\right) \tau \beta\left(y_{1}\right) \tau \beta\left(-y_{2}\right)-\tau \beta\left(y_{1}\right)+\tau \beta\left(y_{2}\right) \\
=\left(-y_{2}\right) \tau \beta\left(y_{1}\right)+\tau \beta\left(-y_{1}\right)=\left(-y_{1}\right) \tau \beta\left(y_{1}\right)+\tau \beta\left(-y_{1}\right) \\
=\tau \beta\left(y_{1}-y_{1}\right)=0 .
\end{gathered}
$$

Thus, $\tau \beta\left(y_{1}-y_{2}\right)=\tau \beta\left(y_{1}\right)-\tau \beta\left(y_{2}\right)$ showing $\tilde{\beta}$ is well-defined.

Suppose that $\sigma_{3} \sigma_{2}(b) \in \operatorname{Im}\left(\sigma_{3} \sigma_{2} \sigma_{1}\right)$, say $a \in A$ with $\sigma_{3} \sigma_{2} b=\sigma_{3} \sigma_{2} \sigma_{1}(a)$. Then $\sigma_{2}(b)-\sigma_{2} \sigma_{1}(a) \in \operatorname{ker}\left(\sigma_{3}=\operatorname{Im} \rho_{2}\right.$, so let $d \in D$ with $\rho_{2}(d)=\sigma_{2}(b)-\sigma_{2} \sigma_{1}(a)$. Thus, $\sigma_{2}(b)=\rho_{2}(d)+\sigma_{2} \sigma_{1}(a)$ and $\rho_{2}(d) \in \operatorname{Im}\left(\sigma_{2}\right) \cap \operatorname{Im}\left(\rho_{2}\right)$. It follows that $\widetilde{\beta} \rho_{2}(d)=$ $\tau \beta \sigma_{2}^{-1}\left(\rho_{2}(d)\right)=\tau \beta\left(b-\sigma_{1}(a)\right)=\tau \beta(b)-\tau \beta\left(\sigma_{1}(a)\right)$. Thus, we will be done by showing that $\tau \beta\left(\sigma_{1}(a) \in \operatorname{Im}(N)\right.$. By part (i), this is equivalent to showing that $\sigma_{2} \sigma_{1}(a) \in \operatorname{Im}\left(\sigma_{2} \sigma_{1}\right)$, which is obviously true.

Conversely, if the class of $\tau \beta(b)$ in $W / \operatorname{Im}(N)$ is in $\widetilde{\beta}\left[\operatorname{Im}\left(\sigma_{2}\right) \cap \operatorname{Im}\left(\rho_{2}\right)\right]$, choose $x \in \operatorname{Im}\left(\sigma_{2}\right) \cap \operatorname{Im}\left(\rho_{2}\right)$ such that $\widetilde{\beta}(x)=\tau \beta(b)+\operatorname{Im}(N)$. Thus, there exists $y \in B$ such that $\sigma_{2}(y)=x$ and $\tau \beta(b)-\tau \beta(y) \in \operatorname{Im}(N)$. By exactness of $A \stackrel{\sigma_{1}}{\rightarrow} B \stackrel{\beta}{\rightarrow} C$ there exists $a \in A$ such that $\sigma_{1}(a)=b-y$, from which it follows that $\sigma_{3} \sigma_{2} \sigma_{1}(a)=\sigma_{3} \sigma_{2}(b)-$ $\sigma_{3} \sigma_{2}(y)=\sigma_{3} \sigma_{2}(b)-\sigma_{3} \sigma_{3}(x)$. But $x \in \operatorname{Im}\left(\rho_{2}\right)=\operatorname{ker}\left(\sigma_{3}\right)$, so $\sigma_{3} \sigma_{2} \sigma_{1}(a)=\sigma_{3} \sigma_{2}(b)$. This completes the proof. 


\section{Controlled homeomorphisms and manifold approximate fi- brations}

In this section we recall the Hughes-Taylor-Williams classification of manifold approximate fibrations, specialize that classification to base spaces $S^{1} \times \mathbb{R}^{i}$, and discuss the relationship with the classical classification of fiber bundles. The main result is Theorem 3.7, which gives necessary and sufficient conditions for a manifold approximate fibration $p: M \rightarrow S^{1}$ with trivial fiber germ to be controlled homeomorphic to a fiber bundle projection (likewise for $p \times \operatorname{id}_{\mathbb{B}}$ ).

Recall that an approximate fibration is a map with the approximate homotopy lifting property. More precisely, we say a map $p: M \rightarrow B$ is an approximate fibration if for every commuting diagram

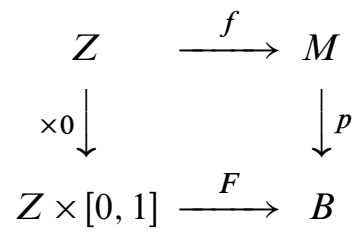

there is a controlled map $\widetilde{F}: Z \times[0,1] \times[0,1) \rightarrow M$ from $F$ to $p$ such that $\widetilde{F}(x, 0, u)=$ $f(x)$ for all $(x, u) \in Z \times[0,1)$. To say $\widetilde{F}$ is a controlled map from $F$ to $p$ means the function $Z \times[0,1] \times[0,1] \rightarrow B$ defined by

$$
(z, t, u) \mapsto \begin{cases}p \tilde{F}(z, t, u) & \text { if } u<1 \\ F(z, t) & \text { if } u=1\end{cases}
$$

is continuous. See Hughes, Taylor and Williams [19, Section 12] for an explanation of how this definition relates to others in the literature.

A proper map $p: M \rightarrow B$ between manifolds (without boundary) is a manifold approximate fibration if $p$ is an approximate fibration.

A controlled homeomorphism between two maps $p: M \rightarrow B$ and $p^{\prime}: M^{\prime} \rightarrow B$ is a one-parameter family $h_{u}: M \rightarrow M^{\prime}, 0 \leq u<1$, of homeomorphisms such that the function $M \times[0,1] \rightarrow B$ defined by

$$
(x, u) \mapsto \begin{cases}p^{\prime} h_{u}(x) & \text { if } u<1 \\ p(x) & \text { if } u=1\end{cases}
$$

is continuous.

Fiber bundles have well-defined fibers up to homeomorphism. Analogously, manifold approximate fibrations have well-defined fiber germs up to controlled homeomorphism 
(see Hughes, Taylor and Williams [19]). Recall that if $p: M \rightarrow B$ is a manifold approximate fibration with $B$ connected, $\operatorname{dim} B=i$ and $\operatorname{dim} M=n \geq 5$, then the fiber germ of $p$ is the manifold approximate fibration $q=p \mid: V=p^{-1}\left(\mathbb{R}^{i}\right) \rightarrow \mathbb{R}^{i}$ where $\mathbb{R}^{i} \hookrightarrow B$ is an open embedding (which is orientation preserving if $B$ is oriented). A trivial fiber germ is the projection $F \times \mathbb{R}^{i} \rightarrow \mathbb{R}^{i}$ for some closed manifold $F$.

In Hughes, Taylor and Williams [19] manifold approximate fibrations over $B$ with total space of dimension greater than four are classified up to controlled homeomorphism. This result is recalled below for the special case $B=S^{1} \times \mathbb{R}^{i}$ and trivial fiber germ, a case that is particularly simple for three reasons. First, the tangent bundle of $B$ plays a role in the classification theorem, but $S^{1} \times \mathbb{R}^{i}$ is parallelizable. Second, the homotopy type of $S^{1} \times \mathbb{R}^{i}$ allows us to view homotopy classes of maps of $S^{1} \times \mathbb{R}^{i}$ into a classifying space of a simplicial group as just the components of the group. Finally, the triviality of the fiber germ can be replaced by bounded homeomorphisms.

First recall the classical classification of fiber bundles over $S^{1} \times \mathbb{R}^{i}$ with fiber $F$. Let $\operatorname{Bun}\left(S^{1} \times \mathbb{R}^{i}\right)_{F}$ denote the simplicial set of fiber bundles over $S^{1} \times \mathbb{R}^{i}$ with fiber $F$, so that there exists a homotopy equivalence $\operatorname{Bun}\left(S^{1} \times \mathbb{R}^{i}\right)_{F} \simeq \operatorname{Map}\left(S^{1}, \operatorname{BTOP}(F)\right)$. Since $\pi_{0} \operatorname{Map}\left(S^{1}, \operatorname{BTOP}(F)\right)=\pi_{0} \operatorname{TOP}(F)$, there is a classifying isomorphism

$$
c_{1}: \pi_{0} \operatorname{Bun}\left(S^{1} \times \mathbb{R}^{i}\right)_{F} \rightarrow \pi_{0} \operatorname{TOP}(F) .
$$

We next recall the classification of manifold approximate fibrations over $S^{1} \times \mathbb{R}^{i}$ with fiber germ $F \times \mathbb{R}^{i+1} \rightarrow \mathbb{R}^{i+1}$. Let $\operatorname{MAF}\left(\mathrm{S}^{1} \times \mathbb{R}^{i}\right)_{F \times \mathbb{R}^{i+1}}$ denote the simplicial set of manifold approximate fibrations over $S^{1} \times \mathbb{R}^{i}$ with fiber germ the projection $F \times \mathbb{R}^{i+1} \rightarrow$ $\mathbb{R}^{i+1}$ and assume $\operatorname{dim} F+i \geq 4$. Since $S^{1} \times \mathbb{R}^{i}$ is parallelizable it follows from Hughes, Taylor and Williams [19] that there is a homotopy equivalence $\operatorname{MAF}\left(\mathrm{S}^{1} \times \mathbb{R}^{i}\right)_{F \times \mathbb{R}^{i+1}} \simeq$ $\operatorname{Map}\left(S^{1}, \operatorname{BTOP}^{\mathrm{c}}\left(F \times \mathbb{R}^{i+1}\right)\right)$ where $\operatorname{TOP}^{\mathrm{c}}\left(F \times \mathbb{R}^{i+1}\right)$ denotes the simplicial group of controlled homeomorphisms on $F \times \mathbb{R}^{i+1}$. Since $\left.\operatorname{TOP}^{\mathrm{c}}\left(F \times \mathbb{R}^{i+1}\right)\right) \simeq \operatorname{TOP}^{\mathrm{b}}(F \times$ $\left.\mathbb{R}^{i+1}\right)$ by Hughes, Taylor and Williams [21] and $\pi_{0} \operatorname{Map}\left(S^{1}, \operatorname{BTOP}^{b}\left(F \times \mathbb{R}^{i+1}\right)\right)=$ $\pi_{0} \operatorname{TOP}^{\mathrm{b}}\left(F \times \mathbb{R}^{i+1}\right)$, there is a classifying isomorphism

$$
c_{2}: \pi_{0} \operatorname{MAF}\left(\mathrm{S}^{1} \times \mathbb{R}^{i}\right)_{F \times \mathbb{R}^{i+1}} \rightarrow \pi_{0} \mathrm{TOP}^{\mathrm{b}}\left(F \times \mathbb{R}^{i+1}\right) .
$$

The next proposition records the relationship between these two classifications.

Proposition 3.1 If $\operatorname{dim} F+i \geq 4$, then the following diagram commutes:

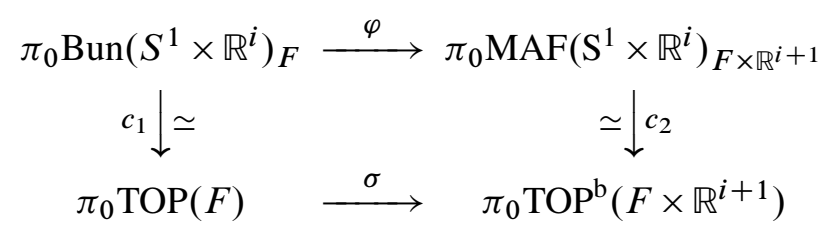


where $\varphi$ is the forgetful map and $\sigma$ is euclidean stabilization $[h] \mapsto\left[h \times \mathrm{id}_{\mathbb{R}^{i+1}}\right]$.

Proof This follows from Hughes, Taylor, and Williams [20, Theorem 0.3].

If $p: M \rightarrow S^{1}$ is a manifold approximate fibration with fiber germ $F \times \mathbb{R} \rightarrow \mathbb{R}$, then the monodromy of $p$ is the class $c_{2}(p)=[h] \in \pi_{0} \mathrm{TOP}^{\mathrm{b}}(F \times \mathbb{R})$ with $h: F \times \mathbb{R} \rightarrow$ $F \times \mathbb{R}$ a bounded homeomorphism. The monodromy induces a well-defined homotopy equivalence $F=F \times\{0\} \stackrel{h \mid}{\rightarrow} F \times \mathbb{R} \rightarrow F$ which in turn induces a homomorphism $h_{*}: \mathrm{Wh}_{1}\left(\mathbb{Z} \pi_{1} F\right) \rightarrow \mathrm{Wh}_{1}\left(\mathbb{Z} \pi_{1} F\right)$, also called the monodromy of $p$.

Theorem 3.2 Let $p: M \rightarrow S^{1}$ be a manifold approximate fibration with fiber germ $F \times \mathbb{R} \rightarrow \mathbb{R}$ and monodromy $[h]$ with $n=\operatorname{dim} F \geq 4$.

(i) The following are equivalent:

(1) $p$ is controlled homeomorphic to a fiber bundle projection with fiber $F$.

(2) $\tau \beta\left(c_{2}([p])\right)=\tau \beta([h])=0 \in \mathrm{Wh}_{1}\left(\mathbb{Z} \pi_{1} F\right)$.

(ii) The following are equivalent:

(1) $\quad p \times \mathrm{id}_{\mathbb{R}}$ is controlled homeomorphic to a fiber bundle projection with fiber $F$.

(2) $\tau \beta\left(c_{2}([p])\right)=\tau \beta([h]) \in \operatorname{Im} N \subseteq \mathrm{Wh}_{1}\left(\mathbb{Z} \pi_{1} F\right)$.

(iii) There exist a subgroup $G$ of $\widetilde{K}_{0}\left(\mathbb{Z} \pi_{1} F\right)$ and a function

$$
N_{0}: G \rightarrow \mathrm{Wh}_{1}\left(\mathbb{Z} \pi_{1} F\right) / \operatorname{Im} N
$$

such that the following are equivalent.

(1) $\quad p \times \mathrm{id}_{\mathbb{R}^{2}}$ is controlled homeomorphic to a fiber bundle projection with fiber $F$.

(2) The class of $\tau \beta\left(c_{2}([p])\right)=\tau \beta([h])$ in $\mathrm{Wh}_{1}\left(\mathbb{Z} \pi_{1} F\right) / \operatorname{Im} N$ is in $N_{0}(G)$.

Proof (i) follows from Proposition 2.3 and Proposition 3.1.

(ii) Consider the diagram

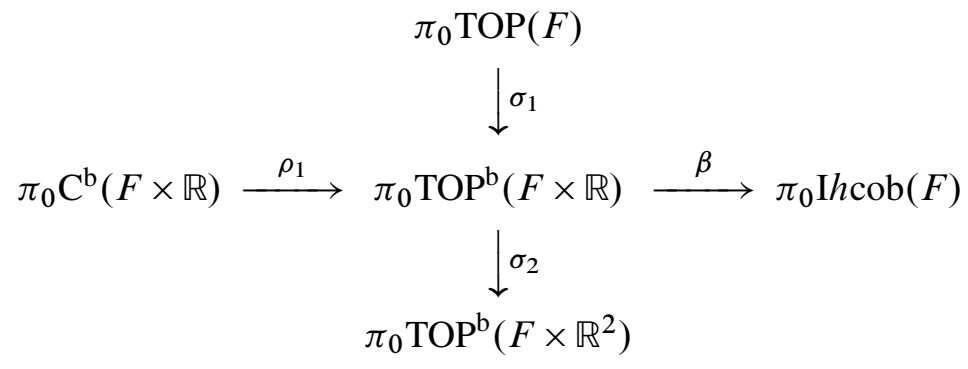


where $\sigma_{1}, \sigma_{2}$ denote euclidean stabilization and $\rho_{1}, \beta$ have been defined above. According to Proposition 3.1, $p \times \mathrm{id}_{\mathbb{R}}$ is controlled homeomorphic to a fiber bundle with fiber $F$ if and only if $\sigma_{2} c_{2}([p])=\sigma_{2}([h]) \in \operatorname{Im}\left(\sigma_{2} \sigma_{1}\right)$. By Propositions 2.1, 2.3, 2.5 and Lemma 2.6, $\sigma_{2}([h]) \in \operatorname{Im}\left(\sigma_{2} \sigma_{1}\right)$ if and only if $\beta([h]) \in \operatorname{Im}\left(\beta \rho_{1}\right)$ if and only if $\tau \beta([h]) \in \operatorname{Im}(N)$. Thus, (1) and (2) are equivalent.

(iii) The diagram above can be extended to a diagram:

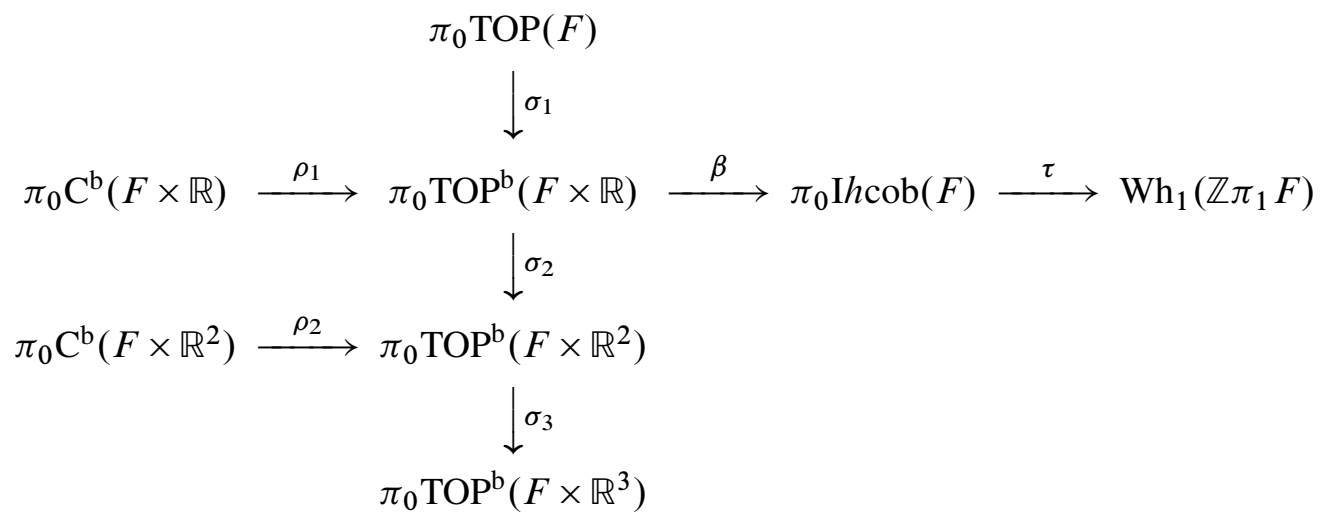

As above, $p \times \mathrm{id}_{\mathbb{R}^{2}}$ is controlled homeomorphic to a fiber bundle projection with fiber $F$ if and only if $\sigma_{3} \sigma_{2}([h]) \in \operatorname{Im}\left(\sigma_{3} \sigma_{2} \sigma_{1}\right)$. Since $\pi_{0} C^{\mathrm{b}}\left(F \times \mathbb{R}^{2}\right) \cong \widetilde{K}_{0}\left(\mathbb{Z} \pi_{1} F\right)$ by Proposition 2.4, the result will follow from Lemma 2.6(ii) once it is observed that the action of $\pi_{0} \mathrm{TOP}^{\mathrm{b}}(F \times \mathbb{R})$ on $\mathrm{Wh}_{1}\left(\mathbb{Z} \pi_{1} F\right)$ satisfies items (3) and (5) of Lemma 2.6(ii). The first follows from the fact that if $\sigma_{2}([h])=\sigma_{2}\left(\left[h^{\prime}\right]\right)$, then the induced homotopy equivalences $h_{1}, h_{1}^{\prime}: F \rightarrow F$ are homotopic and, hence, $h_{1 \#}=h_{1 \sharp}^{\prime}$. The second follows from the explicit construction of $\beta$.

Remark 3.3 It follows from Hughes, Taylor and Williams [22] that Theorem 3.2 condition (i)(1) holds if and only if $p$ is homotopic to a fiber bundle projection with fiber $F$. It seems reasonable to conjecture that Theorem 3.2 condition (ii)(1) holds if and only if $p \times \mathrm{id}_{\mathbb{R}}$ is properly homotopic to a fiber bundle projection with fiber $F$.

We will now prepare for a version of Theorem 3.2(i) and Theorem 3.2(ii) where we allow the fiber of the fiber bundle projections to vary (Theorem 3.7 below). A preliminary result, Lemma 3.5 below, shows that we do not have to worry about non-manifold fibers.

In order to establish Lemma 3.5, we need to use a product formula for Farrell's fibering obstruction $[12 ; 14 ; 13]$. Such a formula seems to be well-known (and at any rate is easy to deduce from the product formula for Whitehead torsion), but a statement does not appear explicitly in the literature. We take this opportunity to 
provide a statement. For notation, suppose $p: M \rightarrow S^{1}$ is a map, where $M$ is a closed, connected manifold, $\operatorname{dim} M \geq 6, p$ induces a surjection $\pi_{1} M \rightarrow \pi_{1} S^{1}$, and the infinite cyclic cover $\bar{M}$ of $M$ is finitely dominated. Farrell's fibering obstruction is an element $\mathcal{F}(p) \in \mathrm{Wh}_{1}\left(\mathbb{Z} \pi_{1} M\right)$ with the property that $\mathcal{F}(p)=0$ if and only if $p$ is homotopic to a fiber bundle projection with manifold fiber. We will use the version of the total fibering obstruction as exposited in Ranicki [33], which we now recall. Let $\zeta: \bar{M} \rightarrow \bar{M}$ be a generating covering translation and let $(d: K \rightarrow \bar{M}, u: \bar{M} \rightarrow K)$ be a finite domination (ie, $K$ is a finite complex and $d u \simeq \mathrm{id}_{\bar{M}}$ ). There is a natural homotopy equivalence given as a composition

$$
h: T(u \zeta d) \rightarrow T(\zeta) \rightarrow M
$$

where $T(\cdot)$ denotes the mapping torus of a map, and the fibering obstruction $\mathcal{F}(p)$ is defined to be the torsion of $h: \mathcal{F}(p):=\tau(p) \in \mathrm{Wh}_{1}\left(\mathbb{Z} \pi_{1} M\right)$.

Lemma 3.4 (Fibering Obstruction Product Formula) Suppose $M$ and $F$ are closed, connected manifolds, $\operatorname{dim} M \geq 6$, there is a map $p: M \rightarrow S^{1}$ inducing a surjection $p_{*}: \pi_{1}(M) \rightarrow \pi_{1}\left(S^{1}\right)$ such that the infinite cyclic cover $\bar{M}$ is finitely dominated, $q$ is the composition $q: M \times F \stackrel{\text { proj }}{\longrightarrow} M \stackrel{p}{\rightarrow} S^{1}$, and $i: M \rightarrow M \times F$ is the inclusion $x \mapsto\left(x, y_{0}\right)$ for a base point point $y_{0} \in F$. Then the fibering obstruction of $q$ is defined and satisfies

$$
\mathcal{F}(q)=\chi(F) i_{*} \mathcal{F}(p) \in \mathrm{Wh}_{1}\left(\mathbb{Z} \pi_{1}(M \times F)\right),
$$

where $\chi(F)$ is the euler characteristic of $F$.

Proof If $(d: K \rightarrow \bar{M}, u: \bar{M} \rightarrow K)$ is a finite domination of $\bar{M}$, then

$$
\left(d \times \operatorname{id}_{F}: K \times F \rightarrow \bar{M} \times F, u \times \operatorname{id}_{F}: \bar{M} \times F \rightarrow K \times F\right)
$$

is a finite domination of the infinite cyclic cover $\bar{M} \times F$ of $M \times F$. Then $\mathcal{F}(q)$ is the torsion of the composition

$$
T(u \zeta d) \times F=T\left((u \zeta d) \times \operatorname{id}_{F}\right) \rightarrow T\left(\zeta \times \operatorname{id}_{F}\right)=T(\zeta) \times F \rightarrow M \times F .
$$

The required formula follows from the product formula for Whitehead torsion due to Kwun and Szczarba [24].

\section{Lemma 3.5}

(i) If $p: M \rightarrow S^{1}$ is a manifold approximate fibration with $m=\operatorname{dim} M \geq 6$ and $p$ is controlled homeomorphic to a bundle projection, then $p$ is controlled homeomorphic to a bundle projection with manifold fiber. 
(ii) If $p: M \rightarrow S^{1} \times \mathbb{R}$ is a manifold approximate fibration with $m=\operatorname{dim} M \geq 7$ and $p$ is controlled homeomorphic to a bundle projection, then $p$ is controlled homeomorphic to a bundle projection with manifold fiber.

\section{Proof}

(i) We may assume that $p: M \rightarrow S^{1}$ is a bundle projection. The fiber is a compact ANR $X$ such that $X \times \mathbb{R}$ is a manifold of dimension 6. According to Hughes, Taylor and Williams [22] it suffices to show that $p$ is homotopic to a bundle projection with manifold fiber; that is, we need to show that the Farrell fibering obstruction $\mathcal{F}(p)$ vanishes. Let $F$ be a closed, connected manifold such that $\chi(F)=1$. Thus, $X \times F$ is a manifold and $q: M \times F \stackrel{\text { proj }}{\rightarrow} M \stackrel{p}{\rightarrow} S^{1}$ is a fiber bundle projection with manifold fiber. In particular, $\mathcal{F}(q)=0$. Since $i_{*}: \mathrm{Wh}_{1}\left(\mathbb{Z} \pi_{1} F\right) \rightarrow \mathrm{Wh}_{1}\left(\mathbb{Z} \pi_{1}(M \times F)\right)$ is an injection, Lemma 3.4 implies that $\mathcal{F}(p)=0$.

(ii) We may assume that $p: M \rightarrow S^{1} \times \mathbb{R}$ is a bundle projection. The fiber is a compact ANR $X$ such that $X \times \mathbb{R}^{2}$ is a manifold of dimension $\geq 7$. Let $W=p^{-1}\left(S^{1} \times\{0\}\right)$ and $q=p \mid: W \rightarrow S^{1} \times\{0\}=S^{1}$. It is unknown whether $X \times \mathbb{R}$ is a manifold (cf Daverman [10, Problem 625]). In particular, $W$ might not be a manifold. However, $W \times \mathbb{R}$ is homeomorphic to $M$ so that $W$ is resolvable by Quinn [31, Corollary 3.2.2]; that is, there exists a manifold $N$, $\operatorname{dim} N=m-1 \geq 6$, and there exists a cell-like map $r: N \rightarrow W$. Let $F$ be a closed, connected manifold such that $\chi(F)=1$. Siebenmann's cell-like approximation theorem [35] theorem implies that there exists a homeomorphism $g: N \times F \rightarrow W \times F$ (since $W \times F$ is a manifold, $g$ arises by approximating $\left.r \times \mathrm{id}_{\mathbb{R}}\right)$. Clearly, the composition $W \times F \stackrel{\text { proj }}{\longrightarrow} W \stackrel{q}{\rightarrow} S^{1}$ is a fiber bundle projection with manifold fiber (the fiber $X \times F$ is a manifold since $X \times \mathbb{R}^{2}$ is a manifold and $\operatorname{dim} F \geq 2$ ). Thus, the composition

$$
q^{\prime}: N \times F \stackrel{g}{\rightarrow} W \times F \stackrel{\text { proj }}{\rightarrow} W \stackrel{q}{\rightarrow} S^{1}
$$

is a fiber bundle projection with manifold fiber. The map $q^{\prime}$ is homotopic to

$$
q^{\prime \prime}: N \times F \stackrel{r \times \operatorname{id}_{F}}{\longrightarrow} W \times F \stackrel{\text { proj }}{\longrightarrow} W \stackrel{q}{\rightarrow} S^{1},
$$

which is seen to be

$$
q^{\prime \prime}: N \times F \stackrel{\text { proj }}{\rightarrow} N \stackrel{r}{\rightarrow} W \stackrel{q}{\rightarrow} S^{1} .
$$

By definition, $\mathcal{F}\left(q^{\prime \prime}\right)=0$. Hence, by using Lemma 3.4 as above, it follows that $\mathcal{F}(q r)=0$. Hence (by Hughes, Taylor and Williams [22]), $q r: N \rightarrow S^{1}$ 
is controlled homeomorphic to a fiber bundle projection $\widetilde{q}: N \rightarrow S^{1}$ with manifold fiber. Since $p: M \rightarrow S^{1} \times \mathbb{R}$ is fiber preserving homeomorphic to $q \times \operatorname{id}_{\mathbb{R}}: W \times \mathbb{R} \rightarrow S^{1} \times \mathbb{R}, p$ is controlled homeomorphic to $q \times \operatorname{id}_{\mathbb{R}}$. Using Siebenmann's theorem [35] again, $r \times \operatorname{id}_{\mathbb{R}}: N \times \mathbb{R} \rightarrow W \times \mathbb{R}$ can be arbitrarily closely approximated by homeomorphisms, so that $q \times \mathrm{id}_{\mathbb{R}}$ is controlled homeomorphic to $q r \times \mathrm{id}_{\mathbb{R}}: N \times \mathbb{R} \rightarrow W \times \mathbb{R}$. Finally, $q \times \mathrm{id}_{\mathbb{R}}$ is controlled homeomorphic to $\widetilde{q} \times \mathrm{id}_{\mathbb{R}}$, which is a bundle projection with manifold fiber.

Lemma 3.6 Let $p: M \rightarrow S^{1}$ be a manifold approximate fibration with fiber germ $F \times \mathbb{R} \rightarrow \mathbb{R}$, monodromy $[h: F \times \mathbb{R} \rightarrow F \times \mathbb{R}]$, and $n=\operatorname{dim} F \geq 4$. Suppose $F^{\prime}$ is a closed manifold for which there is a bounded homeomorphism $k: F \times \mathbb{R} \rightarrow F^{\prime} \times \mathbb{R}$.

(i) $\quad p$ is a manifold approximate fibration with fiber germ $F^{\prime} \times \mathbb{R} \rightarrow \mathbb{R}$ and monodromy $\left[k h k^{-1}: F^{\prime} \times \mathbb{R} \rightarrow F^{\prime} \times \mathbb{R}\right]$.

(ii) If $\beta: \pi_{0} \mathrm{TOP}^{\mathrm{b}}(F \times \mathbb{R}) \rightarrow \pi_{0} \mathrm{I} h \operatorname{cob}(F)$ and $\beta^{\prime}: \pi_{0} \mathrm{TOP}^{\mathrm{b}}\left(F^{\prime} \times \mathbb{R}\right) \rightarrow \pi_{0} \mathrm{I} h \operatorname{cob}\left(F^{\prime}\right)$ are the region between functions defined in section 2 , then there exists $x \in$ $\mathrm{Wh}_{1}\left(\mathbb{Z} \pi_{1} F\right)$ such that

$$
\tau \beta([h])=\left(k^{-1}\right)_{*} \tau \beta^{\prime}\left(\left[k h k^{-1}\right]\right)+x-h_{*}(x)
$$

where $\left(k^{-1}\right)_{*}: \mathrm{Wh}_{1}\left(\mathbb{Z} \pi_{1} F^{\prime}\right) \rightarrow \mathrm{Wh}_{1}\left(\mathbb{Z} \pi_{1} F\right)$ is induced by the composition $F^{\prime}=F^{\prime} \times\{0\} \stackrel{k^{-1} \mid}{\rightarrow} F \times \mathbb{R} \stackrel{F}{\rightarrow}$. Moreover, $x$ is represented by the torsion of the $h$-cobordism associated to the bounded homeomorphism $k^{-1}: F^{\prime} \times \mathbb{R} \rightarrow F \times \mathbb{R}$.

\section{Proof}

(i) If $p$ is considered to have fiber germ $F \times \mathbb{R} \rightarrow \mathbb{R}$, then the affect of the classifying map $c_{2}$ is to turn $p: M \rightarrow S^{1}$ into a fiber bundle over $S^{1}$ with fiber $F \times \mathbb{R}$ and structure group $\operatorname{TOP}^{\mathrm{b}}(F \times \mathbb{R})$. The monodromy $h$ is then the classical monodromy of this bundle. The bundle can be considered to be a bundle with fiber $F^{\prime} \times \mathbb{R}$, structure group $\operatorname{TOP}^{\mathrm{b}}\left(F^{\prime} \times \mathbb{R}\right)$ and monodromy $k h k^{-1}$. See Hughes, Taylor and Williams $[19 ; 20]$.

(ii) Choose $L>0$ large. Let

$$
W=\left(k h k^{-1}\right)\left(F^{\prime} \times(-\infty, L]\right) \backslash F^{\prime} \times(-\infty, 0) \subseteq F^{\prime} \times \mathbb{R}
$$

so that

$$
\left(W ; F^{\prime} \times\{0\}, k h k^{-1}\left(F^{\prime} \times\{L\}\right)\right.
$$

is an $h$-cobordism whose torsion is $\tau \beta^{\prime}\left(\left[k h k^{-1}\right]\right)$. Let

$$
W_{k}=k(F \times[-L, \infty)) \backslash F^{\prime} \times(0, \infty) \subseteq F^{\prime} \times \mathbb{R}
$$


so that $\left(W_{k} ; k(F \times\{-L\}), F^{\prime} \times\{0\}\right)$ is an $h$-cobordism. Let

$$
W_{k^{-1}}=F \times(-\infty, 2 L] \backslash k^{-1}\left(F^{\prime} \times(-\infty, L)\right)
$$

so that $\left(W_{k^{-1}} ; k^{-1}\left(F^{\prime} \times\{L\}\right), F \times\{2 L\}\right)$ is an $h$-cobordism. Let

$$
U=k^{-1} W_{k} \cup k^{-1} W \cup h W_{k^{-1}} \subseteq F \times \mathbb{R} .
$$

Note that $k^{-1} W_{k} \cap k^{-1} W=k^{-1}\left(F^{\prime} \times\{0\}\right), k^{-1} W \cap h W_{k^{-1}}=h k^{-1}\left(F^{\prime} \times\{L\}\right)$, $k^{-1} W_{k} \cap h W_{k^{-1}}=\varnothing$, and that $U=h(F \times(-\infty, 2 L]) \backslash F \times(-\infty,-L) \subseteq$ $F \times \mathbb{R}$ so that $(U ; F \times\{-L\}, h(F \times\{2 L\}))$ is an $h$-cobordism with torsion $\tau(U, F \times\{-L\})=\tau \beta([h]) \in \mathrm{Wh}_{1}\left(\mathbb{Z} \pi_{1} F\right)$. The standard sum and composition formulae imply that

$$
\begin{aligned}
\tau(U, F \times\{-L\})= & \tau\left(k^{-1} W_{k}, F \times\{-L\}\right)+\left(k^{-1}\right)_{*} \tau\left(W, F^{\prime} \times\{0\}\right) \\
& +h_{*}\left(k^{-1}\right)_{*} \tau\left(k W_{k^{-1}}, F^{\prime} \times\{L\}\right) .
\end{aligned}
$$

Let $x=\tau\left(k^{-1} W_{k}, F \times\{-L\}\right) \in \mathrm{Wh}_{1}\left(\mathbb{Z} \pi_{1} F\right)$. It is easy to see that

$$
x+\left(k^{-1}\right)_{*} \tau\left(k W_{k^{-1}}, F^{\prime} \times\{L\}\right)=0
$$

so that $\tau(U, F \times\{-L\})=x+\left(k^{-1}\right)_{*} \tau\left(W, F^{\prime} \times\{0\}\right)-h_{*}(x)$.

Theorem 3.7 Let $p: M \rightarrow S^{1}$ be a manifold approximate fibration with fiber germ $F \times \mathbb{R} \rightarrow \mathbb{R}$ and monodromy $[h]$.

(i) If $n=\operatorname{dim} F \geq 5$, then the following are equivalent.

(a) $p$ is controlled homeomorphic to a fiber bundle projection.

(b) $\tau \beta\left(c_{2}([p])\right)=\tau \beta([h])=0 \in \mathrm{Wh}_{1}\left(\mathbb{Z} \pi_{1} F\right) / \operatorname{Im}\left(1-h_{*}\right)$.

(ii) If $n=\operatorname{dim} F \geq 6$, then the following are equivalent.

(a) $\quad p \times \operatorname{id}_{\mathbb{R}}$ is controlled homeomorphic to a fiber bundle projection.

(b) $\tau \beta\left(c_{2}([p])\right)=\tau \beta([h])=0 \in \mathrm{Wh}_{1}\left(\mathbb{Z} \pi_{1} F\right) /\left(\operatorname{Im} N+\operatorname{Im}\left(1-h_{*}\right)\right)$.

Proof (i) (a) implies (b): By Lemma 3.5(i) we may assume that $p$ is controlled homeomorphic to a bundle projection with fiber a closed manifold $F^{\prime}$. By uniqueness of fiber germs (Hughes, Taylor and Williams [19]) there exists a bounded homeomorphism $k: F \times \mathbb{R} \rightarrow F^{\prime} \times \mathbb{R}$. An application of Theorem 3.2(i) with $F^{\prime}$ replacing $F$ implies that $\tau \beta^{\prime}\left(c_{2}[p]\right)=0 \in \mathrm{Wh}_{1}\left(\mathbb{Z} \pi_{1} F^{\prime}\right)$. Now Lemma 3.6(ii) implies that $\tau \beta\left(c_{2}[p]\right)=$ $\tau \beta([h])=x-h_{*}(x)$ for some $x \in \mathrm{Wh}_{1}\left(\mathbb{Z} \pi_{1} F\right)$.

(b) implies (a): If $\tau \beta([h])=x-h_{*}(x)$ for some $x \in \mathrm{Wh}_{1}\left(\mathbb{Z} \pi_{1} F\right)$, choose an $h-$ cobordism $\left(W ; F, F^{\prime}\right)$ such that $x=\tau(W, F)$. In fact, there is a bounded homeomorphism $k: F \times \mathbb{R} \rightarrow F^{\prime} \times \mathbb{R}$ such that $W=k^{-1}\left(F^{\prime} \times(-\infty, L]\right) \backslash F \times(-\infty, 0)$ 
for some large $L>0$ (this is the $h$-cobordism associated to $k^{-1}$ ). Lemma 3.6(i) implies that $p$ is a manifold approximate fibration with fiber germ $F^{\prime} \times \mathbb{R} \rightarrow \mathbb{R}$ and monodromy $k h k^{-1}$. It follows from Lemma 3.6(ii) that $\left(k^{-1}\right)_{*} \tau \beta^{\prime}\left(\left[k h k^{-1}\right]\right)=0$. Hence $\tau \beta^{\prime}\left(\left[k h k^{-1}\right]\right)=0 \in \mathrm{Wh}_{1}\left(\mathbb{Z} \pi_{1} F^{\prime}\right)$. Finally, Theorem 3.2(i) implies that $p$ is controlled homeomorphic to a bundle projection with fiber $F^{\prime}$.

(ii) (a) implies (b): By Lemma 3.5(ii) we may assume that $p \times \mathrm{id}_{\mathbb{R}}$ is controlled homeomorphic to a bundle projection with fiber a closed manifold $F^{\prime}$. As in (i) there exists a bounded homeomorphism $k: F \times \mathbb{R} \rightarrow F^{\prime} \times \mathbb{R}$. By Lemma 3.6(ii) there exists $x \in \mathrm{Wh}_{1}\left(\mathbb{Z} \pi_{1} F\right)$ such that $\tau \beta([h])=k_{*}^{-1} \tau \beta^{\prime}\left(\left[k h k^{-1}\right]\right)+x-h_{*}(x)$. Lemma 3.6(i) implies that $p$ is a manifold approximate fibration with fiber germ $F^{\prime} \times \mathbb{R} \rightarrow \mathbb{R}$ and monodromy $\left[k h k^{-1}\right]$. Since $p \times \mathrm{id}_{\mathbb{R}}$ is controlled homeomorphic to a fiber bundle projection with fiber $F^{\prime}$, Theorem 3.2 implies that $\tau \beta^{\prime}\left(\left[k h k^{-1}\right]\right)=N^{\prime} z$ for some $z \in \mathrm{Wh}_{1}\left(\mathbb{Z} \pi_{1} F^{\prime}\right)$ where $N^{\prime}: \mathrm{Wh}_{1}\left(\mathbb{Z} \pi_{1} F^{\prime}\right) \rightarrow \mathrm{Wh}_{1}\left(\mathbb{Z} \pi_{1} F^{\prime}\right)$ is the norm map. Thus $\tau \beta([h])=k_{*}^{-1} N^{\prime} z+x-h_{*}(x)=N k_{*}^{-1} z+x-h_{*} x$.

(b) implies (a): Suppose $\tau \beta([h])=N z+x-h_{*} x$. As in (i) there exist a closed manifold $F^{\prime}$ and a bounded homeomorphism $k: F \times \mathbb{R} \rightarrow F^{\prime} \times \mathbb{R}$ such that $x$ is represented by the torsion associated to $k^{-1}$ via the 'region between' construction. Lemma 3.6(ii) implies that $\tau \beta([h])=k_{*}^{-1} \tau \beta^{\prime}\left(\left[k h k^{-1}\right]\right)+x-h_{*} x$. Hence, $k_{*}^{-1} \tau \beta^{\prime}\left(\left[k h k^{-1}\right]\right)=N z$ and $\tau \beta^{\prime}\left(\left[k h k^{-1}\right]\right)=k_{*} N z=N^{\prime} k_{*}^{-1} z$. Since $p$ is a manifold approximate fibration with fiber germ $F^{\prime} \times \mathbb{R} \rightarrow \mathbb{R}$ and monodromy $k h k^{-1}$, Theorem 3.2 implies that $p \times \mathrm{id}_{\mathbb{R}}$ is controlled homeomorphic to a fiber bundle with fiber $F^{\prime}$.

\section{Remark 3.8}

(i) As in Remark 3.3 it follows from Hughes, Taylor and Williams [22] that Theorem 3.7 condition (i)(a) holds if and only if $p$ is homotopic to a fiber bundle projection. It seems reasonable to conjecture that Theorem 3.7 condition (ii)(a) holds if and only if $p \times \mathrm{id}_{\mathbb{R}}$ is properly homotopic to a fiber bundle projection.

(ii) Another way to prove Theorem 3.7(i) is to identify $\tau \beta\left(c_{2}([p])\right)$ with the Farrell fibering obstruction of $p$.

\section{Exotic manifold approximate fibrations}

In this section we combine the results of the previous sections with known $K$-theoretic calculations in order to produce exotic manifold approximate fibrations over $S^{1}$. These are manifold approximate fibrations that are not controlled homeomorphic to fiber bundle projections, even after euclidean stabilization.

Let $\mathbb{Z}_{q}$ denote the finite cyclic group of order $q$. 


\section{Proposition 4.1}

(i) If $\pi_{1}(F)=\mathbb{Z}_{q}, q>3$ is prime, and $\operatorname{dim} F=n \geq 6$ is even, then $N: \mathrm{Wh}_{1}\left(\mathbb{Z}\left[\mathbb{Z}_{q}\right]\right) \rightarrow$ $\mathrm{Wh}_{1}\left(\mathbb{Z}\left[\mathbb{Z}_{q}\right]\right)$ is not surjective, but $\tau \beta: \pi_{0} \mathrm{TOP}^{\mathrm{b}}(F \times \mathbb{R}) \rightarrow \mathrm{Wh}_{1}\left(\mathbb{Z}\left[\mathbb{Z}_{q}\right]\right)$ is surjective.

(ii) If $n \geq 5$ is odd and $q>3$ is prime, then there exists a closed manifold $F$ such that $\operatorname{dim} F=n, \pi_{1}(F)=\mathbb{Z}_{q}$ and $N: \mathrm{Wh}_{1}\left(\mathbb{Z}\left[\mathbb{Z}_{q}\right]\right) \rightarrow \mathrm{Wh}_{1}\left(\mathbb{Z}\left[\mathbb{Z}_{q}\right]\right)$ is not surjective (in fact, it is the 0 homomorphism), but $\tau \beta: \pi_{0} \mathrm{TOP}^{\mathrm{b}}(F \times \mathbb{R}) \rightarrow \mathrm{Wh}_{1}\left(\mathbb{Z}\left[\mathbb{Z}_{q}\right]\right)$ is surjective.

(iii) If $q$ is prime, then $\widetilde{K}_{0}\left(\mathbb{Z}\left[\mathbb{Z}_{q}\right]\right)$ is a finite group and $K_{-i}\left(\mathbb{Z}\left[\mathbb{Z}_{q}\right]\right)=0$ for all $i>0$. If, in addition, $5 \leq q \leq 19$, then $\left.\widetilde{K}_{0}\left(\mathbb{Z}_{\mathbb{Z}}\right]\right)=0$.

Proof Let $q>3$ be a prime number. It is known that $\mathrm{Wh}_{1}\left(\mathbb{Z}\left[\mathbb{Z}_{q}\right]\right)$ is free abelian of finite non-zero rank and the standard involution - acts by the identity (Bass [5], Bass, Milnor and Serre [6], Wall [40]; see Oliver [30, especially Example 1, page 14 and Corollary 7.5] for an exposition). Let $F$ be a closed manifold with $\operatorname{dim} F=n \geq 5$ and $\pi_{1}(F)=\mathbb{Z}_{q}$. Then $N: \mathrm{Wh}_{1}\left(\mathbb{Z} \pi_{1} F\right) \rightarrow \mathrm{Wh}_{1}\left(\mathbb{Z} \pi_{1} F\right)$ is multiplication by 2 if $n$ is even and multiplication by 0 if $n$ is odd, and therefore not surjective. According to Lawson [26, Corollaries 1,2], if $n$ is even, $\pi_{0} \mathrm{I} h \operatorname{cob}(F)=\pi_{0} h \operatorname{cob}(F)$, and if $n$ is odd, there exist manifolds $F$ as above such that $\pi_{0} \mathrm{I} h \operatorname{cob}(F)=\pi_{0} h \operatorname{cob}(F)$. Since Proposition 2.3 implies that $\beta: \pi_{0} \mathrm{TOP}^{\mathrm{b}}(F \times \mathbb{R}) \rightarrow \pi_{0} \mathrm{I} h \operatorname{cob}(F)=\pi_{0} h \operatorname{cob}(F)$ is surjective, it follows that $\tau \beta: \pi_{0} \mathrm{TOP}^{\mathrm{b}}(F \times \mathbb{R}) \rightarrow \mathrm{Wh}_{1}\left(\mathbb{Z}\left[\mathbb{Z}_{q}\right]\right)$ is surjective. This proves (i) and (ii).

For (iii) see Rosenberg [34, pages 23, 157].

\section{Theorem 4.2}

(i) If $q$ is a prime, $5 \leq q \leq 19, n \geq 6$ is even, $F$ is any closed manifold with $\pi_{1}(F)=\mathbb{Z}_{q}$ and $\operatorname{dim} F=n$, then there exists a manifold approximate fibration $p: M \rightarrow S^{1}$ with fiber germ $F \times \mathbb{R} \rightarrow \mathbb{R}$ such that for all $i \geq 0, p \times \operatorname{id}_{\mathbb{R}^{i}}: M \times$ $\mathbb{R}^{i} \rightarrow S^{1} \times \mathbb{R}^{i}$ is not controlled homeomorphic to a fiber bundle projection with fiber $F$.

(ii) If $q>3$ is prime, $n \geq 5$ is odd, then there exists a closed manifold $F$ with $\pi_{1}(F)=\mathbb{Z}_{q}$ and $\operatorname{dim} F=n$ and a manifold approximate fibration $p: M \rightarrow S^{1}$ with fiber germ $F \times \mathbb{R} \rightarrow \mathbb{R}$ such that for all $i \geq 0, p \times \mathrm{id}_{\mathbb{R}^{i}}: M \times \mathbb{R}^{i} \rightarrow S^{1} \times \mathbb{R}^{i}$ is not controlled homeomorphic to a fiber bundle projection with fiber $F$.

(iii) If $n \geq 6$ is even, $F$ is any closed manifold with $\pi_{1}(F)=\mathbb{Z}_{5}$ and $\operatorname{dim} F=n$, then there exists a manifold approximate fibration $p: M \rightarrow S^{1}$ with fiber germ $F \times \mathbb{R} \rightarrow \mathbb{R}$ such that for all $i \geq 0, p \times \mathrm{id}_{\mathbb{R}^{i}}: M \times \mathbb{R}^{i} \rightarrow S^{1} \times \mathbb{R}^{i}$ is not controlled homeomorphic to a fiber bundle projection. 
(iv) If $n \geq 5$ is odd, then there exists a closed manifold $F$ with $\pi_{1}(F)=\mathbb{Z}_{5}$ and $\operatorname{dim} F=n$ and a manifold approximate fibration $p: M \rightarrow S^{1}$ with fiber germ $F \times \mathbb{R} \rightarrow \mathbb{R}$ such that for all $i \geq 0, p \times \mathrm{id}_{\mathbb{R}^{i}}: M \times \mathbb{R}^{i} \rightarrow S^{1} \times \mathbb{R}^{i}$ is not controlled homeomorphic to a fiber bundle projection.

\section{Proof}

(i) According to Proposition 3.1 we need a manifold approximate fibration $p: M \rightarrow$ $S^{1}$ with fiber germ $F \times \mathbb{R} \rightarrow \mathbb{R}$ and monodromy $[h] \in \pi_{0} \mathrm{TOP}^{\mathrm{b}}(F \times \mathbb{R})$ such that for all $i \geq 0,\left[h \times \mathrm{id}_{\mathbb{R}^{i}}\right] \in \pi_{0} \operatorname{TOP}^{\mathrm{b}}\left(F \times \mathbb{R}^{i+1}\right)$ is not in $\operatorname{Im}\left(\sigma: \pi_{0} \operatorname{TOP}(F) \rightarrow\right.$ $\left.\pi_{0} \operatorname{TOP}^{\mathrm{b}}\left(F \times \mathbb{R}^{i+1}\right)\right)$. According to Proposition 2.1, Proposition 2.4, and Proposition 4.1(iii), $\sigma: \pi_{0} \mathrm{TOP}^{\mathrm{b}}\left(F \times \mathbb{R}^{2+i}\right) \rightarrow \pi_{0} \mathrm{TOP}^{\mathrm{b}}\left(F \times \mathbb{R}^{3+i}\right)$ is injective for all $i \geq 0$. Hence, it suffices to find a manifold approximate fibration $p$ with monodromy $[h] \in \pi_{0} \operatorname{TOP}^{\mathrm{b}}(F \times \mathbb{R})$ such that $\left[h \times \mathrm{id}_{\mathbb{R}}\right] \in \pi_{0} \mathrm{TOP}^{\mathrm{b}}\left(F \times \mathbb{R}^{2}\right)$ is not in $\operatorname{Im}\left(\sigma: \pi_{0} \operatorname{TOP}(F) \rightarrow \pi_{0} \mathrm{TOP}^{\mathrm{b}}\left(F \times \mathbb{R}^{2}\right)\right)$; that is, such that $p \times \mathrm{id}_{\mathbb{R}}$ is not controlled homeomorphic to a fiber bundle projection with fiber $F$. According to Theorem 3.2(ii) this is equivalent to $\tau \beta([h]) \neq 0 \in \mathrm{Wh}_{1}\left(\mathbb{Z} \pi_{1} F\right) / \operatorname{Im} N$. Such monodromies exist by Proposition 4.1(i).

(ii) This is similar to (i) except now we know only that $\sigma: \pi_{0} \mathrm{TOP}^{\mathrm{b}}\left(F \times \mathbb{R}^{3+i}\right) \rightarrow$ $\pi_{0} \operatorname{TOP}^{\mathrm{b}}\left(F \times \mathbb{R}^{4+i}\right)$ is injective for all $i \geq 0$. Hence, it suffices to find a manifold approximate fibration $p$ with monodromy $[h] \in \pi_{0} \mathrm{TOP}^{\mathrm{b}}(F \times \mathbb{R})$ such that $\left[h \times \mathrm{id}_{\mathbb{R}^{2}}\right] \in \pi_{0} \operatorname{TOP}^{\mathrm{b}}\left(F \times \mathbb{R}^{3}\right)$ is not in $\operatorname{Im}\left(\sigma: \pi_{0} \mathrm{TOP}(F) \rightarrow \pi_{0} \mathrm{TOP}^{\mathrm{b}}\left(F \times \mathbb{R}^{3}\right)\right)$; that is, such that $p \times \mathrm{id}_{\mathbb{R}^{2}}$ is not controlled homeomorphic to a fiber bundle projection with fiber $F$. According to Proposition 4.1(ii),(iii) $\mathrm{Wh}_{1}\left(\mathbb{Z} \pi_{1} F\right) / \operatorname{Im} N=$ $\mathrm{Wh}_{1}\left(\mathbb{Z} \pi_{1} F\right)$ and is infinite (cf proof of Proposition 4.1). Hence, since Proposition 4.1(iii) implies that $\widetilde{K}_{0}\left(\mathbb{Z}\left[\mathbb{Z}_{q}\right]\right)$ is finite, the result follows from Theorem 3.2(iii).

(iii) As in (i) it suffices to find a manifold approximate fibration $p: M \rightarrow S^{1}$ with fiber germ $F \times \mathbb{R} \rightarrow \mathbb{R}$ and monodromy $[h] \in \pi_{0} \mathrm{TOP}^{\mathrm{b}}(F \times \mathbb{R})$ such that $p \times \mathrm{id}_{\mathbb{R}}$ is not controlled homeomorphic to a fiber bundle projection. According to Theorem 3.7(ii) this is equivalent to $\tau \beta([h]) \neq 0 \in \mathrm{Wh}_{1}\left(\mathbb{Z} \pi_{1} F\right) /(\operatorname{Im} N+\operatorname{Im}(1-$ $\left.h_{*}\right)$ ). But $\mathrm{Wh}_{1}\left(\mathbb{Z} \pi_{1} F\right)=\mathrm{Wh}_{1}\left(\mathbb{Z}\left[\mathbb{Z}_{5}\right]\right)$ is isomorphic to $\mathbb{Z}$ so that $h_{*}= \pm 1$ and $1-h_{*}=0,2$. As noted in the proof of Proposition 4.1, $N=0$ so that $\mathrm{Wh}_{1}\left(\mathbb{Z} \pi_{1} F\right) /\left(\operatorname{Im} N+\operatorname{Im}\left(1-h_{*}\right)\right) \neq 0$ and the result follows from Theorem 3.7(ii).

(iv) is similar to (iii). 


\section{Neighborhood germ classification and proof of the Main Theorem}

In this section we recall the classification of neighborhood germs of manifold stratified pairs given by Hughes, Taylor, Weinberger and Williams [18]. We then combine this neighborhood germ classification with the results on manifold approximate fibrations given in Section 4 to prove the Main Theorem of Section 1.

We begin by giving the definition of the special type of stratified pairs that appear in the Main Theorem, namely, the locally conelike manifold stratified pairs.

Let $(X, A)$ be a pair of spaces so that $A \subseteq X$. Then $X$ is said to have two strata: the lower (or bottom) stratum $A$ and the top stratum $X \backslash A$.

Definition 5.1 A pair $(X, A)$ is a locally conelike manifold stratified pair provided:

(1) $X$ is a separable metric space and $A$ is a closed subspace of $X$.

(2) $A$ and $X \backslash A$ are topological manifolds with $i=\operatorname{dim} A$.

(3) For each $x \in A$ there exists a closed manifold $F_{x}$ and an open embedding $\mathbb{R}^{i} \times \stackrel{\circ}{\mathrm{c}} F_{X} \rightarrow X$ such that $(0, v) \mapsto x$ and $\mathbb{R}^{i} \times\{v\}$ is mapped into $A$. Here, $\stackrel{\circ}{\mathrm{c}} F_{X}$ is the open cone on $F_{X}$ and $v$ is the cone point.

Locally conelike manifold stratified pairs are precisely the locally conelike TOP stratified sets of Siebenmann [36] with two strata.

The main source of locally conelike manifold stratified for us arise as follows. Let $p: M \rightarrow B$ be a manifold approximate fibration with trivial fiber germ $F \times \mathbb{R}^{i} \rightarrow \mathbb{R}^{i}$, and let $X=\operatorname{cyl}(p)$, the open mapping cylinder of $p$. Then $(X, B)$ is a locally conelike manifold stratified pair. For if $x \in B$ and $\mathbb{R}^{i}$ is an euclidean neighborhood of $x$ (with 0 corresponding to $x$ ), then $p \mid: p^{-1}\left(\mathbb{R}^{i}\right) \rightarrow \mathbb{R}^{i}$ is controlled homeomorphic to the projection $p_{2}: F \times \mathbb{R}^{i} \rightarrow \mathbb{R}^{i}$. Thus, there is a homeomorphism $h: \operatorname{cyl}\left(p_{2}\right) \rightarrow \operatorname{cyl}(p \mid)$ that preserves the levels of the mapping cylinders and restricts to the identity of $\mathbb{R}^{i}$ (see Hughes, Taylor, Weinberger and Williams [18, Proposition 3.16]). Since $\operatorname{cyl}\left(p_{2}\right)=\stackrel{\circ}{\mathrm{c}}(F) \times \mathbb{R}^{i}$ and $\stackrel{\circ}{\operatorname{cyl}}(p \mid)$ is an open neighborhood of $x$ in $X$, this shows that $X$ is locally conelike.

Even though we are interested here in locally conelike stratified pairs, the classification theorem of Hughes, Taylor, Weinberger and Williams [18] takes place in the more general setting of manifold stratified pairs. The relevant concepts can be found in Quinn [32] and Weinberger [42] as well as [18], but we collect here the necessary definitions for the convenience of the reader. 
If $(X, A)$ and $(Y, B)$ are two pairs, then a map $f:(X, A) \rightarrow(Y, B)$ is said to be strict, or stratum-preserving, if $f(X \backslash A) \subseteq Y \backslash B$ and $f(A) \subseteq B$. The subspace $A$ of $X$ is said to be forward tame if there exists a neighborhood $N$ of $A$ in $X$ and a strict map $H:(N \times I, A \times I \cup N \times\{0\}) \rightarrow(X, A)$ such that $H(x, t)=x$ for all $(x, t) \in A \times I$ and $H(x, 1)=x$ for all $x \in N$.

Let $\operatorname{Map}_{\mathrm{s}}((X, A),(Y, B))$ denote the space of strict maps with the compact-open topology. The homotopy link of $A$ in $X$ is

$$
\operatorname{holink}(X, A)=\operatorname{Map}_{\mathrm{s}}(([0,1],\{0\}),(X, A)) .
$$

Evaluation at 0 defines a map $q$ : holink $(X, A) \rightarrow A$ which should be thought of as a model for a normal fibration of $A$ in $X$.

The pair $(X, A)$ is said to be a homotopically stratified pair if $A$ is forward tame in $X$ and if $q$ : holink $(X, A) \rightarrow A$ is a fibration. If in addition, the fiber of $q: \operatorname{holink}(X, A) \rightarrow$ $A$ is finitely dominated, then $(X, A)$ is said to be homotopically stratified with finitely dominated local holinks. (When we say that the fiber of $q$ is finitely dominated and $A$ is not path connected, we mean that each fiber of $q$ is finitely dominated.) If the strata $A$ and $X \backslash A$ are manifolds (without boundary), $X$ is a locally compact separable metric space, and $(X, A)$ is homotopically stratified with finitely dominated local holinks, then $(X, A)$ is a manifold stratified pair.

We now define the set of equivalence classes of neighborhoods that is the subject of the classification theorem of Hughes, Taylor, Weinberger and Williams [18] recalled below. Let $B$ be an $i$-manifold (without boundary) and let $n \geq 0$ be a fixed integer. A germ of a stratified neighborhood of $B$ is an equivalence class represented by a manifold stratified pair $(X, B)$ with $\operatorname{dim}(X \backslash B)=n$. Two such pairs $(X, B)$ and $(Y, B)$ are germ equivalent provided that there exist open neighborhoods $U$ and $V$ of $B$ in $X$ and $Y$, respectively, and a homeomorphism $h: U \rightarrow V$ such that $h \mid B=\operatorname{id}_{B}$.

Let $p: X \rightarrow Y \times \mathbb{R}$ be a map. The teardrop of $p$, denoted by $X \cup_{p} Y$, is defined to be the space with underlying set the disjoint union $X \amalg Y$ and topology given as follows. First, let $c: X \cup_{p} Y \rightarrow Y \times(-\infty,+\infty]$ be defined by

$$
c(x)= \begin{cases}p(x) & \text { if } x \in X \\ (x,+\infty) & \text { if } x \in Y .\end{cases}
$$

Then the topology on $X \cup_{p} Y$ is the minimal topology such that

(i) $X \subseteq X \cup_{p} Y$ is an open embedding, and

(ii) $c$ is continuous. 
Here is the main result from Hughes, Taylor, Weinberger and Williams [18].

Theorem 5.2 (Neighborhood Germ Classification [18, page 876]) Let $n \geq 5$ and let $B$ be a closed manifold. The teardrop construction defines a bijection from the set of controlled homeomorphism classes of manifold approximate fibrations over $B \times \mathbb{R}$ (with total space of dimension $n$ ) to the set of germs of stratified neighborhoods of $B$ (with top stratum of dimension $n$ ).

We can now give the proof of the Main Theorem (which we first restate) of Section 1.

Main Theorem For every integer $m \geq 6$ there exists a locally conelike manifold stratified pair $\left(X, S^{1}\right)$ with $\operatorname{dim}\left(X \backslash S^{1}\right)=m$ such that $S^{1}$ has a manifold approximate fibration mapping cylinder neighborhood in $X$, but for each $i \geq 0 S^{1} \times T^{i}$ does not have a fiber bundle mapping cylinder neighborhood in $X \times T^{i}$. In fact, $S^{1} \times T^{i}$ does not have a block bundle mapping cylinder neighborhood in $X \times T^{i}$.

Here, $T^{i}$ denotes the $i$-dimensional torus, $T^{i}=S^{1} \times \cdots S^{1}$ ( $i$ times).

Proof of Main Theorem Let $X$ be the open mapping cylinder cyl $(p)$ of a manifold approximate fibration $p: M \rightarrow S^{1}$ constructed in Theorem 4.2(iii) or (iv). Since $p$ has trivial fiber germ, it follows from the remarks above that $\left(X, S^{1}\right)$ is a locally conelike manifold stratified pair.

Suppose there exists $i \geq 0$ such that $S^{1} \times T^{i}$ had a fiber bundle mapping cylinder neighborhood in $X \times T^{i}$. Then there are a closed manifold $N$ and a fiber bundle projection $q: N \rightarrow S^{1} \times T^{i}$ such that $\left(X \times T^{i}, S^{1} \times T^{i}\right)$ and $\left(\operatorname{cyl}(q), S^{1} \times T^{i}\right)$ are germ equivalent. In other words, the teardrops

$$
\left(\left(M \times T^{i} \times \mathbb{R}\right) \cup_{\left(p \times \operatorname{id}_{T^{i}} \times \operatorname{id}_{\mathbb{R}}\right)}\left(S^{1} \times T^{i}\right), S^{1} \times T^{i}\right)
$$

and

$$
\left((N \times \mathbb{R}) \cup_{\left(q \times \mathrm{id}_{\mathbb{R}}\right)}\left(S^{1} \times T^{i}\right), S^{1} \times T^{i}\right)
$$

are germ equivalent. It follows from Theorem 5.2 that $p \times \mathrm{id}_{T^{i}} \times \mathrm{id}_{\mathbb{R}}: M \times T^{i} \times \mathbb{R} \rightarrow$ $S^{1} \times T^{i} \times \mathbb{R}$ and $q \times \operatorname{id}_{\mathbb{R}}: N \times \mathbb{R} \rightarrow S^{1} \times T^{i} \times \mathbb{R}$ are controlled homeomorphic. Form the pull-back:

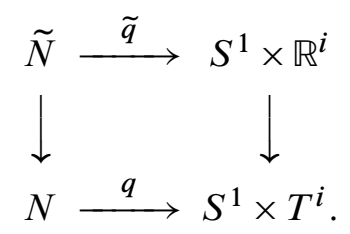


It follows from Lemma 5.3 below that $p \times \operatorname{id}_{\mathbb{R}^{i+1}}: M \times \mathbb{R}^{i+1} \rightarrow S^{1} \times \mathbb{R}^{i+1}$ and $\tilde{q} \times \operatorname{id}_{\mathbb{R}}: \tilde{N} \times \mathbb{R} \rightarrow S^{1} \times \mathbb{R}^{i+1}$ are controlled homeomorphic. Since $\tilde{q} \times \operatorname{id}_{\mathbb{R}}$ is a fiber bundle projection, this contradicts the choice of $p$. Hence, $S^{1} \times T^{i}$ does not have a fiber bundle mapping cylinder neighborhood in $X \times T^{i}$.

Since block bundles with fiber $F$ are classified by $\widetilde{\mathrm{BTOP}}(F)$, equivalence classes of block bundles over $S^{1} \times \mathbb{R}^{i}$ correspond to $\pi_{0} \widetilde{\mathrm{TOP}}(F)$. Since $\pi_{0} \mathrm{TOP}(F) \rightarrow$ $\pi_{0} \widetilde{\mathrm{TOP}}(F)$ is surjective, the result on block bundles follows from the fiber bundle case.

The following is an elementary lemma used in the proof above.

Lemma 5.3 Suppose $p: M \rightarrow B, q: N \rightarrow B$ are two maps, $\alpha: \widetilde{B} \rightarrow B$ is a covering projection and there are two pull-back diagrams:
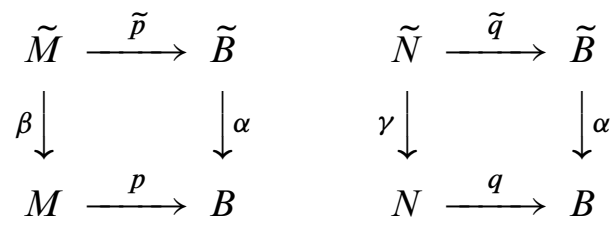

If $p$ and $q$ are controlled homeomorphic, then so are $\tilde{p}$ and $\tilde{q}$.

Proof Let $h_{t}: M \rightarrow N, 0 \leq t<1$, be a controlled homeomorphism from $p$ to $q$. Thus, $M \times[0,1] \rightarrow B$ defined by $(x, t) \mapsto\left\{\begin{array}{ll}q h_{t}(x) & \text { if } 0 \leq t<1 \\ p(x) & \text { if } t=1\end{array}\right.$ is continuous. Define $g: \tilde{M} \times[0,1] \rightarrow B$ by $(x, t) \mapsto\left\{\begin{array}{ll}q h_{t} \beta(x) & \text { if } 0 \leq t<1 \\ p \beta(x) & \text { if } t=1\end{array}\right.$. Thus, $g$ is continuous. Since $p \beta=\alpha \tilde{p}$, there exists (uniquely) a map $\tilde{g}: \tilde{M} \times[0,1] \rightarrow \widetilde{B}$ such that $\widetilde{g}_{1}=\tilde{\tilde{p}}$ and $\alpha \widetilde{g}_{t}=g_{t}$ for all $t \in[0,1]$. Thus, it is possible to define $\widetilde{h}_{t}: \tilde{M} \rightarrow \tilde{N} \subseteq N \times \widetilde{B}$ by $\tilde{h}_{t}(x)=\left(h_{t} \beta(x), \tilde{g}_{t}(x)\right)$ for $0 \leq t<1$. One can check that $\tilde{h}_{t}$ is a controlled homeomorphism from $\tilde{p}$ to $\tilde{q}$.

\section{Remark 5.4}

(i) If $(X, B)$ is a manifold stratified pair, it is the case that for a torus of sufficiently high dimension, the quotient $X \times T /(B \times T=B)$ does have a block structure. Moreover, the block structure on the "links" is not arbitrary: it has some nice transfer invariance properties. In other words, for each simplex $\Delta$ of $B$ one has a nice manifold which maps to $\Delta \times T$, with control in the $T$ direction. (What we have shown here is that one cannot block over simplices of $B \times T$.) This 
structure is called a $\mathrm{STIBB}^{1}$ in Weinberger [42] and is applied there to give a stable surgery exact sequence for stratified spaces. Indeed, if one had block structures stably then the $L$-cosheaves in the stable classification theorem [42, Section 6.2] would have to have the " $s$ " decoration (as in the "PT category" in [42, Section 6.1]) rather than the $-\infty$ decoration that arises. The differences between these decorations are accounted for by Tate cohomology calculations rather similar to those done here.

It is not too difficult to combine Theorem 5.2 with the classification theorem of Hughes-Taylor-Williams [19], and the stabilization theorem of Weiss-Williams [43] to give a proof of the stable classification theorem for $S^{-\infty}(X$ rel $B)$. Using [19] the stable germ neighborhoods are computed by maps $\left[B, \operatorname{BTOP}^{\mathrm{b}}(F \times E)\right]$ which is the same as $\left[B, \widetilde{\mathrm{BTOP}}^{\mathrm{b}}(F \times E)\right]$ by [43], the last of which is computed by bounded block surgery using $L^{-\infty}$ (holink). Different structures with the same germ near the singular stratum can then be compared using ordinary rel $\infty$ surgery on the complement. The result of this analysis is just a Poincaré duality away from the result as expressed in Weinberger [42].

(ii) These examples are closely related to those constructed by Anderson [1]. In fact, the examples in [1] are locally conelike manifold stratified pairs $\left(X, S^{1}\right)$ such that $S^{1}$ has a manifold approximate fibration mapping cylinder neighborhood in $X$, but $S^{1}$ does not have a fiber bundle mapping cylinder neighborhood in $X$. Anderson was not concerned with the stability issues addressed here.

(iii) Husch [23] used nontrivial inertial $h$-cobordisms to construct exotic manifold approximate fibrations over $S^{1}$. In fact, our Theorem 3.7(i) is just a precise formulation of the analysis in [23]. In connection with this, it should be pointed out that a manifold approximate fibration $p: M \rightarrow S^{1}$ with $\operatorname{dim} M \geq 5$ is controlled homeomorphic to a fiber bundle projection if and only if $p$ is homotopic to a fiber bundle projection by Hughes, Taylor and Williams [22].

(iv) Ferry and Pedersen [15] construct interesting embeddings of $S^{1}$ in $S^{n}$. However, their examples $\left(S^{n}, S^{1}\right)$ need not be locally conelike manifold stratified pairs.

(v) Using the tables for relative class numbers in Washington [41, page 412], it is possible to construct a few more even dimensional manifolds as in Theorem 4.2(i) for primes $q$ with $3<q<67$. We do not know of other calculations that give more manifolds as in Theorem 4.2(iii) and (iv).

\footnotetext{
${ }^{1}$ An equivalent notion is used by Yan [45]: one has blocks over $\Delta \times E$ where $E$ is an Euclidean space, and the data is bounded in the $E$ direction.
} 


\section{References}

[1] D R Anderson, A triangulable space which possesses no natural piecewise linear structure, Topology 17 (1978) 297-302 MR508893

[2] D R Anderson, W C Hsiang, Extending combinatorial PL structures on stratified spaces, Invent. Math. 32 (1976) 179-204 MR0413114

[3] D R Anderson, W C Hsiang, The functors $K_{-1}$ and pseudo-isotopies of polyhedra, Ann. of Math. (2) 105 (1977) 201-223 MR0440573

[4] D R Anderson, W C Hsiang, Extending combinatorial piecewise linear structures on stratified spaces. II, Trans. Amer. Math. Soc. 260 (1980) 223-253 MR570787

[5] H Bass, K-theory and stable algebra, Inst. Hautes Études Sci. Publ. Math. (1964) 5-60 MR0174604

[6] H Bass, J Milnor, J-P Serre, Solution of the congruence subgroup problem for $\mathrm{SL}_{n}(n \geq 3)$ and $\mathrm{Sp}_{2 n}(n \geq 2)$, Inst. Hautes Études Sci. Publ. Math. 33 (1967) 59137 MR0244257

[7] W Browder, F Quinn, A surgery theory for $G$-manifolds and stratified sets, from: “Manifolds-Tokyo 1973 (Proc. Internat. Conf., Tokyo, 1973)”, Univ. Tokyo Press, Tokyo (1975) 27-36 MR0375348

[8] S Cappell, J L Shaneson, Nonlinear similarity, Ann. of Math. (2) 113 (1981) 315-355 MR607895

[9] S Cappell, S Weinberger, A simple construction of Atiyah-Singer classes and piecewise linear transformation groups, J. Differential Geom. 33 (1991) 731-742 MR1100209

[10] R J Daverman, Problems about finite-dimensional manifolds, from: "Open problems in topology”, North-Holland, Amsterdam (1990) 431-455 MR1078662

[11] R D Edwards, R C Kirby, Deformations of spaces of imbeddings, Ann. Math. (2) 93 (1971) 63-88 MR0283802

[12] F T Farrell, The obstruction to fibering a manifold over a circle, Bull. Amer. Math. Soc. 73 (1967) 737-740 MR0215310

[13] F T Farrell, The obstruction to fibering a manifold over a circle, from: "Actes du Congrès International des Mathématiciens (Nice, 1970), Tome 2", Gauthier-Villars, Paris (1971) 69-72 MR0423376

[14] F T Farrell, The obstruction to fibering a manifold over a circle, Indiana Univ. Math. J. 21 (1971/1972) 315-346 MR0290397

[15] S C Ferry, E K Pedersen, Some mildly wild circles in $S^{n}$ arising from algebraic K-theory, K-Theory 4 (1991) 479-499 MR1116929

[16] J-C Hausmann, $h$-cobordismes entre variétés homéomorphes, Comment. Math. Helv. 50 (1975) 9-13 MR0368037 
[17] B Hughes, Bounded homotopy equivalences of Hilbert cube manifolds, Trans. Amer. Math. Soc. 287 (1985) 621-643 MR768729

[18] B Hughes, L R Taylor, S Weinberger, B Williams, Neighborhoods in stratified spaces with two strata, Topology 39 (2000) 873-919 MR1763954

[19] B Hughes, L R Taylor, B Williams, Bundle theories for topological manifolds, Trans. Amer. Math. Soc. 319 (1990) 1-65 MR1010410

[20] B Hughes, L R Taylor, B Williams, Manifold approximate fibrations are approximately bundles, Forum Math. 3 (1991) 309-325 MR1115949

[21] B Hughes, L R Taylor, B Williams, Bounded homeomorphisms over Hadamard manifolds, Math. Scand. 73 (1993) 161-176 MR1269255

[22] B Hughes, L R Taylor, B Williams, Rigidity of fibrations over nonpositively curved manifolds, Topology 34 (1995) 565-574 MR1341809

[23] L S Husch, Approximating approximate fibrations by fibrations, Canad. J. Math. 29 (1977) 897-913 MR0500990

[24] K W Kwun, R H Szczarba, Product and sum theorems for Whitehead torsion, Ann. of Math. (2) 82 (1965) 183-190 MR0182972

[25] R Lashof, M Rothenberg, G-smoothing theory, from: "Algebraic and geometric topology (Proc. Sympos. Pure Math., Stanford Univ., Stanford, CA, 1976), Part 1", Proc. Sympos. Pure Math., XXXII, Amer. Math. Soc., Providence, R.I. (1978) 211-266 MR520506

[26] T C Lawson, Inertial h-cobordisms with finite cyclic fundamental group, Proc. Amer. Math. Soc. 44 (1974) 492-496 MR0358820

[27] W Ling, Translations on $M \times \mathbb{R}$, from: "Algebraic and geometric topology (Proc. Sympos. Pure Math., Stanford Univ., Stanford, CA, 1976), Part 2”, Proc. Sympos. Pure Math., XXXII, Amer. Math. Soc., Providence, R.I. (1978) 167-180 MR520533

[28] J N Mather, Notes on topological stability, Harvard Univ., Cambridge, photocopied (1970)

[29] J Milnor, Whitehead torsion, Bull. Amer. Math. Soc. 72 (1966) 358-426 MR0196736

[30] R Oliver, Whitehead groups of finite groups, London Mathematical Society Lecture Note Series 132, Cambridge University Press, Cambridge (1988) MR933091

[31] F Quinn, Ends of maps. I, Ann. of Math. (2) 110 (1979) 275-331 MR549490

[32] F Quinn, Homotopically stratified sets, J. Amer. Math. Soc. 1 (1988) 441-499 MR928266

[33] A Ranicki, Lower $K$ - and L-theory, London Mathematical Society Lecture Note Series 178, Cambridge University Press, Cambridge (1992) MR1208729

[34] J Rosenberg, Algebraic $K$-theory and its applications, Graduate Texts in Mathematics 147, Springer, New York (1994) MR1282290 
[35] L C Siebenmann, Approximating cellular maps by homeomorphisms, Topology 11 (1972) 271-294 MR0295365

[36] L C Siebenmann, Deformation of homeomorphisms on stratified sets. I, II, Comment. Math. Helv. 47 (1972) 123-136; ibid. 47 (1972), 137-163 MR0319207

[37] L Siebenmann, J Sondow, Some homeomorphic sphere pairs that are combinatorially distinct, Comment. Math. Helv. 41 (1966/1967) 261-272 MR0214082

[38] R Thom, Ensembles et morphismes stratifiés, Bull. Amer. Math. Soc. 75 (1969) 240284 MR0239613

[39] F Waldhausen, Algebraic $K$-theory of spaces, a manifold approach, from: "Current trends in algebraic topology, Part 1 (London, Ont., 1981)", CMS Conf. Proc. 2, Amer. Math. Soc., Providence, R.I. (1982) 141-184 MR686115

[40] C T C Wall, Norms of units in group rings, Proc. London Math. Soc. (3) 29 (1974) 593-632 MR0376746

[41] L C Washington, Introduction to cyclotomic fields, second edition, Graduate Texts in Mathematics 83, Springer, New York (1997) MR1421575

[42] S Weinberger, The topological classification of stratified spaces, Chicago Lectures in Mathematics, University of Chicago Press, Chicago, IL (1994) MR1308714

[43] M Weiss, B Williams, Automorphisms of manifolds and algebraic $K$-theory. I, KTheory 1 (1988) 575-626 MR953917

[44] H Whitney, Local properties of analytic varieties, from: "Differential and Combinatorial Topology (A Symposium in Honor of Marston Morse)", Princeton Univ. Press, Princeton, NJ (1965) 205-244 MR0188486

[45] M Yan, The periodicity in stable equivariant surgery, Comm. Pure Appl. Math. 46 (1993) 1013-1040 MR1223661

BH: Department of Mathematics, Vanderbilt University,

Nashville TN 37240, USA

LRT, BW: Department of Mathematics, University of Notre Dame,

Notre Dame IN 46556, USA

SW: Department of Mathematics, University of Chicago,

Chicago IL 60637, USA

bruce.hughes@vanderbilt.edu, taylor.2@nd.edu,

shmuel@math.uchicago.edu, williams.4@nd.edu

http://www.math.vanderbilt.edu/ hughescb/, http://www.nd.edu/ taylor/, http://math.uchicago.edu/ shmuel/, http://www.nd.edu/ bruce/

Proposed: Steve Ferry

Seconded: Rob Kirby and Wolfgang Lueck
Received: 19th November 2003

Revised: 31 May 2007 